\title{
Extensive transcriptomic study emphasizes importance of vesicular transport in C9orf72 expansion carriers
}

Dennis W. Dickson ${ }^{1 \dagger}$, Matthew C. Baker ${ }^{1 \dagger}$, Jazmyne L. Jackson ${ }^{1 \dagger}$, Mariely DeJesus-Hernandez ${ }^{1}$, NiCole A. Finch ${ }^{1}$, Shulan Tian², Michael G. Heckman³, Cyril Pottier', Tania F. Gendron', Melissa E. Murray', Yingxue Ren", Joseph S. Reddy ${ }^{4}$, Neill R. Graff-Radford ${ }^{5}$, Bradley F. Boeve ${ }^{6}$, Ronald C. Petersen ${ }^{6}$, David S. Knopman ${ }^{6}$, Keith A. Josephs ${ }^{6}$, Leonard Petrucelli ${ }^{1}$, Björn Oskarsson ${ }^{5}$, John W. Sheppard ${ }^{7}$, Yan W. Asmann ${ }^{4}$, Rosa Rademakers ${ }^{*^{*}}$ and Marka van Blitterswijk ${ }^{* *}$

\begin{abstract}
The majority of the clinico-pathological variability observed in patients harboring a repeat expansion in the C9orf72-SMCR8 complex subunit (C9orf72) remains unexplained. This expansion, which represents the most common genetic cause of frontotemporal lobar degeneration (FTLD) and motor neuron disease (MND), results in a loss of C9orf72 expression and the generation of RNA foci and dipeptide repeat (DPR) proteins. The C9orf72 protein itself plays a role in vesicular transport, serving as a guanine nucleotide exchange factor that regulates GTPases. To further elucidate the mechanisms underlying C9orf72-related diseases and to identify potential disease modifiers, we performed an extensive RNA sequencing study. We included individuals for whom frontal cortex tissue was available: FTLD and FTLD/MND patients with $(n=34)$ or without $(n=44)$ an expanded C9orf72 repeat as well as control subjects $(n=24)$. In total, 6706 genes were differentially expressed between these groups (false discovery rate $[F D R]<0.05$ ). The top gene was C9orf72 (FDR $=1.41 \mathrm{E}-14$ ), which was roughly two-fold lower in C9orf72 expansion carriers than in (disease) controls. Coexpression analysis revealed groups of correlated genes (modules) that were enriched for processes such as protein folding, RNA splicing, synaptic signaling, metabolism, and Golgi vesicle transport. Within our cohort of C9orf72 expansion carriers, machine learning uncovered interesting candidates associated with clinico-pathological features, including age at onset (vascular endothelial growth factor A [VEGFA]), C9orf72 expansion size (cyclin dependent kinase like 1 [CDKL 1]), DPR protein levels (eukaryotic elongation factor 2 kinase [EEF2K]), and survival after onset (small G protein signaling modulator 3 [SGSM3]). Given the fact that we detected a module involved in vesicular transport in addition to a GTPase activator (SGSM3) as a potential modifier, our findings seem to suggest that the presence of a C9orf72 repeat expansion might hamper vesicular transport and that genes affecting this process may modify the phenotype of C9orf72-linked diseases.
\end{abstract}

Keywords: Frontotemporal dementia, Frontotemporal lobar degeneration, Amyotrophic lateral sclerosis, Motor neuron disease, C9orf72, Transcriptomics, Vesicular transport, Machine learning, RNA sequencing, Repeat expansion disorders

\footnotetext{
* Correspondence: Rademakers.Rosa@mayo.edu;

VanBlitterswijk.Marka@mayo.edu

${ }^{\dagger}$ Dennis W. Dickson, Matthew C. Baker and Jazmyne L. Jackson shared first authorship.

${ }^{\dagger}$ Rosa Rademakers and Marka van Blitterswijk shared last authorship.

'Department of Neuroscience, Mayo Clinic, 4500 San Pablo Road,

Jacksonville, FL 32224, USA

Full list of author information is available at the end of the article
}

(c) The Author(s). 2019 Open Access This article is distributed under the terms of the Creative Commons Attribution 4.0 International License (http://creativecommons.org/licenses/by/4.0/), which permits unrestricted use, distribution, and reproduction in any medium, provided you give appropriate credit to the original author(s) and the source, provide a link to the Creative Commons license, and indicate if changes were made. The Creative Commons Public Domain Dedication waiver (http://creativecommons.org/publicdomain/zero/1.0/) applies to the data made available in this article, unless otherwise stated. 


\section{Introduction}

Substantial clinical and pathological variability has been reported in patients carrying an expanded repeat in the C9orf72-SMCR8 complex subunit (C9orf72) [58], which leads to frontotemporal dementia (FTD) and amyotrophic lateral sclerosis (ALS) $[14,50]$. While FTD is the second most frequent cause of dementia in the presenile group, ALS is the most common form of motor neuron disease (MND). Intriguingly, there is considerable clinical, genetic, and pathological overlap between FTD and ALS. In fact, up to $40 \%$ of FTD patients demonstrate motor neuron involvement $[7,44]$. Similarly, up to $50 \%$ of ALS patients have cognitive impairment and 15\% fulfill the FTD criteria $[17,46]$. Mutations in several genes appear to be specific for either FTD or ALS (e.g., superoxide dismutase 1 [SOD1]); however, most have been detected in both diseases, like the repeat expansion in C9orf72. Furthermore, TAR DNA-binding protein 43 (TDP-43) inclusions can be observed in approximately $50 \%$ of FTD patients and more than $90 \%$ of ALS patients $[43,44]$. Given this overlap, FTD and ALS are thought to represent a disease spectrum.

The repeat expansion in C9orf72 accounts for about $30 \%$ of familial cases and $5-10 \%$ of sporadic cases [41, 58], possibly due to a reduction in C9orf72 expression [14], the aggregation of flawed RNA transcripts in the nucleus of cells (RNA foci) [14], and the formation of repetitive proteins aberrantly translated from the expansion (dipeptide repeat [DPR] proteins) $[4,42]$. The C9orf72 protein itself is known to interact with endosomes and functions in vesicle trafficking $[18,56]$.

Thus far, a limited number of studies has been performed to investigate the expression pattern of C9orf72linked diseases. We have, for instance, profiled brain tissue of C9orf72 expansion carriers using expression arrays, which uncovered an upregulation of transthyretin and homeobox genes [19]. In an RNA sequencing study, we also examined differential expression, alternative splicing, and alternative polyadenylation in ALS patients harboring a C9orf72 expansion [47]. We detected widespread transcriptome changes in the cerebellum, particularly of RNAprocessing events [47]. Furthermore, we observed elevated levels of repetitive elements (e.g., long interspersed nuclear elements [LINEs]) in patients with a C9orf72 repeat expansion [48]. Several other studies also investigated expression patterns distinctive of an expanded repeat in C9orf72 by examination of laser-captured motor neurons, lymphoblastoid cell lines, fibroblast and induced pluripotent stem cell (iPSC) lines, iPSC-derived motor neuron cultures, and/or postmortem motor cortex tissue from C9orf72 expansion carriers [11, 16, 30, 52, 54].

Despite these efforts, the majority of the clinicopathological variability remains unexplained in $C 9$ orf 72 expansion carriers. As such, we have performed an in- depth RNA sequencing study on frontal cortex tissue from a well-characterized cohort. We evaluated individuals who received a pathological diagnosis of frontotemporal lobar degeneration (FTLD) with or without MND as well as control subjects stored at the Mayo Clinic Florida Brain Bank $(n=102)$. In addition to differential expression and co-expression analyses, we used various analytical approaches within the group of C9orf72 expansion carriers to identify genes associated with clinical and pathological features of C9orf72-related diseases. Our findings provide additional evidence for the involvement of vesicle-mediated transport and reveal several potential modifiers of C9orf72linked diseases.

\section{Materials and methods \\ Subjects}

Subjects were selected for whom frozen brain tissue was available in our Mayo Clinic Florida Brain Bank $(n=102$; Table 1). Frontal cortex tissue was collected from the middle frontal gyrus at the level of the nucleus accumbens. We included C9orf72 expansion carriers $(n=34)$ pathologically diagnosed with FTLD characterized by TDP-43 inclusions (FTLD-TDP) in the presence or absence of MND, patients with FTLD-TDP or FTLD/MND without known mutations (type A or B; $n=44$ ), and control subjects without neurological diseases $(n=24)$. Our C9orf72 expansion carriers had a median age at death of 69 years (interquartile range [IQR]: 62-76), a median RNA integrity number (RIN) of 8.9 (IQR: 8.4-9.5), and $35 \%$ was female. For patients without a repeat expansion, the median age at death was 78 years (IQR: 68-83), their median RIN value was 9.6 (IQR: 9.1-9.8), and 50\% was female. The median age at death of control subjects was 87 years (IQR: 78-89) with a median RIN value of 9.1 (IQR: $8.8-9.6)$ and $67 \%$ was female. Of note, in previous studies, we already obtained the expansion size, RNA foci burden, and DPR protein levels for the majority of our expansion carriers [13, 21, 57]. Methylation levels of the C9orf72 promoter were determined using $100 \mathrm{ng}$ of DNA as input material with a quantitative methylationsensitive restriction enzyme-based assay, as described elsewhere $[40,51]$.

\section{RNA sequencing}

Total RNA was extracted from frozen brain tissue using the RNeasy Plus Mini Kit (Qiagen). RNA quality and quantity were determined with a 2100 Bioanalyzer Instrument (Agilent) using the RNA Nano Chip (Agilent); only samples with a RIN value above 7.0 were included. Libraries were made using the TruSeq RNA Library Prep Kit (Illumina; v2) and sequenced at 10 samples/lane as paired-end 101 base-pair reads on a HiSeq 4000 (Illumina) at Mayo Clinic's Genome Analysis Core. Subsequently, raw sequencing reads were aligned to the human reference 
Table 1 Subject characteristics

\begin{tabular}{llll}
\hline Variable & C9Plus $(n=34)$ & C9Minus $(n=44)$ & Control $(n=24)$ \\
\hline Age at death (years) & $69.0(62.0-75.8)$ & $78.0(67.8-83.2)$ & $86.5(78.2-89.2)$ \\
RIN (value) & $8.9(8.4-9.5)$ & $9.6(9.1-9.8)$ & $9.1(8.8-9.6)$ \\
Sex (female) & $12(35 \%)$ & $22(50 \%)$ & $16(67 \%)$ \\
Diagnosis (FTLD/MND) & $12(35 \%)$ & $13(30 \%)$ & $0(0 \%)$ \\
\hline
\end{tabular}

Data are sample median (interquartile range [IQR]) or number (\%). Information is shown for patients carrying a C9orf72 repeat expansion (C9Plus), patients without this repeat expansion (C9Minus), and control subjects without neurological diseases (Control). Age at death, RNA integrity number (RIN), sex, and pathological diagnosis (frontotemporal lobar degeneration [FTLD] with motor neuron disease [MND]) are specified

genome (GRCh38) with Spliced Transcripts Alignment to a Reference (STAR; v2.5.2b) [15]. After alignment, library quality was assessed using RSeQC (v3.0.0) [60], and genelevel expression was quantified using the Subread package (v1.5.1) [37]. All analyses described below were performed in $\mathrm{R}$ ( $\mathrm{R}$ Core Team; v3.5.3).

\section{Differential expression analysis}

We used conditional quantile normalization (CQN) to account for differences in gene counts, gene lengths, and GC content, resulting in comparable quantile-by-quantile distributions across samples [24, 49]. Genes were kept if their maximum normalized and log2-transformed reads per kb per million (RPKM) values were above zero $(n=$ 24,092). Using linear regression models, source of variation (SOV) analysis was then performed to determine how much variation was explained by the disease group (C9orf72 expansion carriers, non-expansion carriers, and controls) as well as by potential confounders (RIN, sex, age at death, plate, and gene counts). We also assessed the effects of differences in cellular composition between individuals using surrogate markers for five major cell types: neurons (enolase 2 [ENO2]), microglia (CD68 molecule $[C D 68]$ ), astrocytes (glial fibrillary acidic protein [GFAP]), oligodendrocytes (oligodendrocyte transcription factor 2 [OLIG2]), and endothelial cells (CD34 molecule [CD34]) $[1,12,23]$. Based on our SOV analysis, variables with a mean F-statistic above 1.25 were selected. Differential expression analysis was performed using two separate linear regression models: one model included RIN, sex, age at death, plate, and disease group, while the other model also included our five surrogate markers for the major cell types. Fold-changes were determined and $p$-values were adjusted for multiple testing using a false discovery rate (FDR) procedure [5]. Genes with an FDR below 5\% were considered statistically significant $($ FDR $<0.05)$. To examine whether significantly differentially expressed genes were enriched for biological processes and pathways, enrichment analysis was performed using the anRichment package [33] and gene sets from the molecular signatures database (MSigDB; v6.2) [39]. For visualization purposes, Venn diagrams were generated with the VennDiagram package [10]. Moreover, heat maps were made with the ComplexHeatmap package [22] and the flashClust package [35], utilizing the Euclidean distance and average method.

\section{Co-expression analysis}

In addition to the gene-level analyses described in the previous section, we performed module-level analyses to identify the building blocks of biological systems, revealing relevant information about the system's structure and dynamics as well as the function of certain proteins [61]. As such, we employed weighted gene co-expression network analysis (WGCNA) to find modules comprised of highly correlated genes that go up or down together [34], using residual expression values adjusted for aforementioned potential confounders as input (both with and without surrogate markers). Separate analyses were performed for each pairwise comparison, creating signed hybrid networks and using the biweight midcorrelation (bicor) method. To achieve a scale-free topology, we selected a power appropriate for each comparison, ranging between 4 and 14. A dynamic tree cutting method was used with a minimum module size of 30 and a merge height varying from 0.25 to 0.35 , depending on the comparison. Modules generated using these settings were represented by their first principal component (module eigengene) and a unique color. For every gene, we calculated correlations between expression levels and each module's eigengene value (module membership). Modules that differed significantly between disease groups were further investigated using enrichment analyses and displayed with heat maps, using methods identical to those described above. Additionally, network visualization was performed for top protein-coding genes belonging to modules of interest with a relatively high module membership (>0.6), utilizing the force-directed yFiles Organic Layout and Organic Edge Router algorithms in Cytoscape (v3.7.1) [55]. In these network plots, the connectivity of each gene was represented by the size of its node, the module to which it has been assigned by its color, and the strength of the correlation by the thickness of its edges.

\section{Clinico-pathological association analysis}

To find associations with clinical and pathological features of the disease in patients carrying an expanded 
C9orf72 repeat $(n=34)$, we obtained residuals from linear regression models with expression levels as outcome to account for potential confounders (RIN, sex, and plate, either with or without surrogate markers). First, we performed analyses to examine individual genes, starting with linear regression models. We investigated associations with age at onset and age at death, adjusting for disease subgroup (FTLD or FTLD/MND). Subsequently, we assessed associations with C9orf72 expansion size, RNA foci burden (mean percentage of cells with sense or antisense RNA foci), DPR protein levels (total poly[GP]), and methylation of the C9orf 72 promoter, while adjusting for disease subgroup and age at death. Hereafter, we performed a logistic regression analysis to compare expression levels between patients with predominant FTLD to those diagnosed with both FTLD and MND, adjusting for age at death. We ran Cox proportional hazard regression models, including disease subgroup and age at death as potential confounders. Hazard ratios (HRs) and 95\% confidence intervals (CIs) were estimated; deaths of any cause were utilized as our survival endpoint. Three approaches were used for our survival analysis to assess expression levels: comparing the top $50 \%$ to the bottom $50 \%$ as a dichotomous categorical variable, ranking expression levels from low to high, and examining them as a continuous variable. Notably, all models were adjusted for multiple testing using an FDR procedure [5]; an FDR below 5\% was considered statistically significant $(\mathrm{FDR}<0.05)$.

Second, we evaluated combinations of genes found to be nominally significant in our single-gene analysis $(P<$ $0.05)$. To examine the sensitivity of our results, we opted to use two machine learning methods, namely Least Absolute Shrinkage and Selection Operator (LASSO) regression and random forest. LASSO regression was performed with the glmnet package [20]. The most parsimonious model was selected, using leave-one-out cross-validation, an alpha of one, and a lambda within one standard error from the model with the lowest cross-validation error (mean squared error, classification error, or partial-likelihood deviance). This approach was employed using models appropriate for the nature of the given response variable, including age at onset, age at death, expansion size, RNA foci burden, poly(GP) DPR levels, $C 9$ orf 72 promoter methylation, disease subgroup, and survival after onset. We then used the randomForest package [38], which implements Breiman's random forest algorithm [6]. We tuned the number of trees in the forest $(1000$ to 30,000$)$, the number of features considered at each split (2 to 98 ), and the size of terminal nodes (2 to 10). Subsequently, we created a random forest regressor (age at onset, age at death, C9orf 72 expansion size, RNA foci levels, DPR proteins, and promoter methylation) or classifier (disease subgroup). We extracted the out-of-bag error rate as well as information about the importance of each gene (variable importance), as represented by its permuted effect on the error rate (e.g., mean squared error or accuracy), while other genes remained unchanged [38].

\section{Validation experiments and analysis}

We validated RNA expression levels of the top candidate genes in C9orf72 expansion carriers from our RNA sequencing cohort $(n=34)$. Reverse transcription was performed using $250 \mathrm{ng}$ of RNA as template with the SuperScript III Kit (Invitrogen) and an equal ratio of Random Hexamers and Oligo dT primers. The following expression assays (TaqMan) were performed: vascular endothelial growth factor A (VEGFA; Hs00900055_m1), cyclin dependent kinase like 1 (CDKL1; Hs01012519_m1), eukaryotic elongation factor 2 kinase (EEF2K; Hs00179434_ $\mathrm{m} 1$ ), and small G protein signaling modulator 3 (SGSM3; Hs00924186_g1). As markers, ENO2 (Hs00157360_m1) and GFAP (Hs00909233_m1) were selected. To obtain relative expression levels for each patient, the median of replicates was taken, the geometric mean of the two markers was calculated, and a calibrator on every plate was used for normalization, utilizing the $\Delta \Delta \mathrm{Ct}$ method. Subsequently, the correlation between these relative expression levels and residuals from our RNA sequencing analysis was calculated using a Spearman's test of correlation.

\section{Results}

\section{Top differentially expressed gene is C9orf72}

We performed RNA sequencing on carriers of a C9orf72 repeat expansion $(\mathrm{n}=34)$, FTLD and FTLD/MND patients without this expansion $(n=44)$, and control subjects without any neurological disease $(n=24$; Table 1$)$. When adjusting for cell-type-specific markers, 6706 genes were significantly different between these groups. Without adjustment, 11,770 genes were differentially expressed. Importantly, the top gene was C9orf72 itself, both with $(\mathrm{FDR}=1.41 \mathrm{E}-14)$ and without $(\mathrm{FDR}=8.69 \mathrm{E}$ 08) adjustment for cell-type-specific markers (Table 2; Fig. 1a, b). Hereafter, we specifically compared patients with a C9orf72 expansion to patients without this expansion or to controls. For simplicity, we focused on results that accounted for differences in cellular composition. In total, we detected 4443 differentially expressed genes when comparing expansion carriers to patients without this expansion and 2334 genes when comparing them to controls (Fig. 1c). Heat maps demonstrated that most patients with an expanded repeat clustered together (Fig. 2), especially when comparing them to controls. Of the differentially expressed genes, 1460 overlapped (Fig. 1c, d), including C9orf72 itself. The RNA expression levels of $C 9$ orf72 were roughly two-fold lower in expansion carriers than in non-expansion carriers (FDR $=6.04 \mathrm{E}-06)$ or 
Table 2 Differential Expression (All Groups)

\begin{tabular}{|c|c|c|c|c|c|c|c|c|}
\hline \multirow[t]{2}{*}{ Order } & \multicolumn{4}{|c|}{ With Cell-Type-Specific Markers } & \multicolumn{4}{|c|}{ Without Cell-Type-Specific Markers } \\
\hline & Gene & Chr & $P$-value & FDR & Gene & Chr & $P$-value & FDR \\
\hline 1 & C9orf72 & chr9 & $5.86 \mathrm{E}-19$ & $1.41 \mathrm{E}-14$ & C9orf72 & chr9 & $3.61 \mathrm{E}-12$ & $8.69 \mathrm{E}-08$ \\
\hline 2 & SMIM14 & chr4 & $2.06 \mathrm{E}-11$ & 2.49E-07 & RP11-196E1.3 & chr11 & $3.89 \mathrm{E}-11$ & $4.68 \mathrm{E}-07$ \\
\hline 3 & FBLL1 & chr5 & $1.12 \mathrm{E}-10$ & 8.96E-07 & GEM & chr8 & $3.45 \mathrm{E}-10$ & 2.05E-06 \\
\hline 4 & $A B C E 1$ & chr4 & $3.54 \mathrm{E}-10$ & 2.13E-06 & PCP4 & chr21 & $3.47 \mathrm{E}-10$ & 2.05E-06 \\
\hline 5 & $\mathrm{HRH3}$ & chr20 & $9.60 \mathrm{E}-10$ & 4.62E-06 & KCNC3 & chr19 & $5.85 \mathrm{E}-10$ & 2.05E-06 \\
\hline 6 & GIT1 & chr17 & 1.41E-09 & 5.55E-06 & ANGPT1 & chr8 & $6.33 \mathrm{E}-10$ & 2.05E-06 \\
\hline 7 & AC009133.15 & chr16 & 1.61E-09 & 5.55E-06 & SMIM14 & chr4 & $6.85 \mathrm{E}-10$ & 2.05E-06 \\
\hline 8 & PYCR2 & chr1 & 2.33E-09 & $6.24 \mathrm{E}-06$ & GPCPD1 & chr20 & 7.04E-10 & 2.05E-06 \\
\hline 9 & VSTM2A-OT1 & chr7 & $2.52 \mathrm{E}-09$ & $6.24 \mathrm{E}-06$ & KREMEN2 & chr16 & $8.08 \mathrm{E}-10$ & 2.05E-06 \\
\hline 10 & MTR & chr1 & 2.59E-09 & $6.24 \mathrm{E}-06$ & DPYSL3 & chr5 & 8.49E-10 & 2.05E-06 \\
\hline 11 & KCNJ6 & chr21 & 3.06E-09 & $6.26 \mathrm{E}-06$ & SCNIB & chr19 & $9.75 \mathrm{E}-10$ & 2.14E-06 \\
\hline 12 & RP11-196E1.3 & chr11 & 3.26E-09 & $6.26 \mathrm{E}-06$ & PRELP & chr1 & $1.38 \mathrm{E}-09$ & $2.78 \mathrm{E}-06$ \\
\hline 13 & TESK1 & chr9 & 3.98E-09 & $6.26 \mathrm{E}-06$ & FBLL 1 & chr5 & $1.58 \mathrm{E}-09$ & 2.93E-06 \\
\hline 14 & DCAF16 & chr4 & 4.07E-09 & $6.26 \mathrm{E}-06$ & LINC01102 & chr2 & 1.95E-09 & 3.17E-06 \\
\hline 15 & SH3GL1P3 & chr17 & 5.26E-09 & $6.26 \mathrm{E}-06$ & CTD-2126E3.4 & chr19 & 1.98E-09 & 3.17E-06 \\
\hline 16 & DCTNG & chr8 & 5.26E-09 & $6.26 \mathrm{E}-06$ & SLC17A6 & chr11 & 2.10E-09 & 3.17E-06 \\
\hline 17 & $B P G M$ & chr7 & 5.57E-09 & $6.26 \mathrm{E}-06$ & $R A B 4 B$ & chr19 & 2.23E-09 & 3.17E-06 \\
\hline 18 & NT5DC1 & chr6 & 5.58E-09 & $6.26 \mathrm{E}-06$ & KB-1107E3.1 & chr8 & 3.56E-09 & 4.77E-06 \\
\hline 19 & RP11-318A15.7 & chr17 & 5.59E-09 & $6.26 \mathrm{E}-06$ & CCDC102A & chr16 & 4.10E-09 & 5.19E-06 \\
\hline 20 & CHMP2B & chr3 & 5.69E-09 & $6.26 \mathrm{E}-06$ & $A E B P 1$ & chr7 & 4.62E-09 & 5.39E-06 \\
\hline
\end{tabular}

Differentially expressed genes are displayed either with or without adjustment for cell-type-specific markers. For each gene, the chromosome (Chr), $p$-value, and false discovery rate (FDR) are included

control subjects (FDR $=1.08 \mathrm{E}-05$; Table 3 ). We further investigated overlapping genes using enrichment analyses, which indicated that these genes might be enriched for processes involved in endocytosis (FDR $=0.02$; Table 4).

\section{Co-expression analysis reveals relevant modules involved in processes like vesicular transport}

Next, we performed module-level analyses using WGCNA. When comparing patients with an expanded C9orf72 repeat to those without this repeat, we identified 22 modules. Visualization of the module-trait relationships (Fig. 3a), revealed that the strongest relationships were dependent on the presence or absence of a C9orf72 repeat expansion (disease group). In fact, we only detected significant correlations with the disease group, resulting in the identification of 11 modules of interest. None of these modules demonstrated a significant correlation with potential confounders, such as cellular composition, RIN, age at death, sex, or plate (Fig. 3a). Enrichment analysis of these 11 modules (Table 5) showed that they were involved in protein folding (black), RNA splicing (blue), metabolic processes (yellow), Golgi vesicle transport (green), GABAergic interneuron differentiation (greenyellow), synaptic signaling (turquoise), etc. Given the potential function of the C9orf72 protein, we visualized the green module (Fig. 4a); most expansion carriers appeared to have lower module eigengene values for this module than disease controls. In addition to Golgi vesicle transport $(\mathrm{FDR}=1.33 \mathrm{E}-06)$, the green module was also significantly enriched for related processes, such as endoplasmic reticulum to Golgi vesicle-mediated transport $(\mathrm{FDR}=1.97 \mathrm{E}-05)$, vacuolar transport $\quad(\mathrm{FDR}=9.91 \mathrm{E}-05)$, vesicle-mediated transport $(\mathrm{FDR}=0.002)$, and lysosomes $(\mathrm{FDR}=0.002)$. This is in agreement with the cellular components that appeared to be involved, including vacuolar part $(\mathrm{FDR}=4.31 \mathrm{E}-10)$, endoplasmic reticulum part $(\mathrm{FDR}=$ $2.88 \mathrm{E}-09)$, endoplasmic reticulum $(\mathrm{FDR}=2.34 \mathrm{E}-08)$, vacuole $(\mathrm{FDR}=8.41 \mathrm{E}-08)$, and vacuolar membrane $(\mathrm{FDR}=$ 6.53E-07). A gene network, which displayed top genes from significant modules, demonstrated that members of the green module (e.g., charged multivesicular body protein $2 \mathrm{~B}$ $[C H M P 2 B]$ ) clustered together with genes belonging to the yellow module, most importantly C9orf72 (Fig. 5a).

The comparison between expansion carriers and controls resulted in 25 modules. Despite the fact that we adjusted for cell-type-specific markers and other potential confounders, we still observed weak correlations with those variables; for instance, due to differences in cellular composition between affected and unaffected frontal cortices (Fig. 3b). Nevertheless, the disease group displayed the strongest correlations and was significantly 


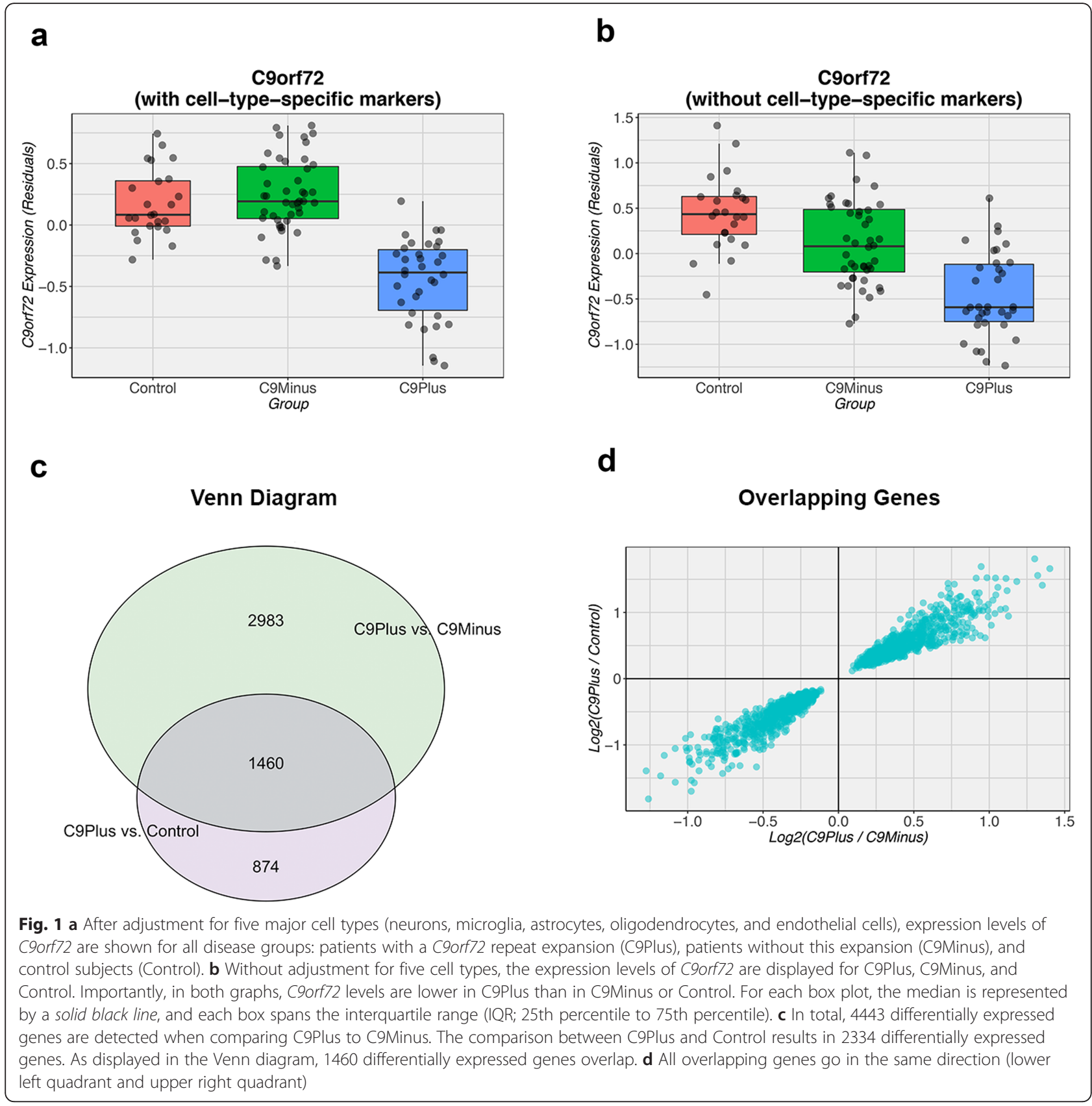

associated with 11 modules. An enrichment was seen for processes like GABAergic interneuron differentiation (paleturquoise), synaptic signaling (turquoise), metabolic processes (yellow), Golgi vesicle transport (green), oxidative phosphorylation (orange), protein folding (midnightblue), and cell death (steelblue; Table 6). The C9orf72 gene was assigned to the yellow module, which we visualized (Fig. 4b); in general, expansion carriers seemed to have decreased module eigengene values for the yellow module, when comparing them to control subjects. The yellow module was enriched for various processes, including small-molecule metabolic processes (FDR $=2.10 \mathrm{E}-13)$, organic-acid catabolic processes (FDR $=1.39 \mathrm{E}-11)$, smallmolecule catabolic processes (FDR $=1.15 \mathrm{E}-10)$, organicacid metabolic processes (FDR $=6.24 \mathrm{E}-08)$, and oxidation reduction processes $(\mathrm{FDR}=8.71 \mathrm{E}-07)$. The top cellular components were the mitochondrial matrix $(\mathrm{FDR}=2.59 \mathrm{E}-10)$, mitochondrion $(\mathrm{FDR}=2.18 \mathrm{E}-09)$, and mitochondrial part $(\mathrm{FDR}=2.27 \mathrm{E}-09)$. Our gene network with top genes from significant modules highlighted genes belonging to the yellow module (Fig. 5b), such as small integral membrane protein 14 (SMIM14), pyrroline- 

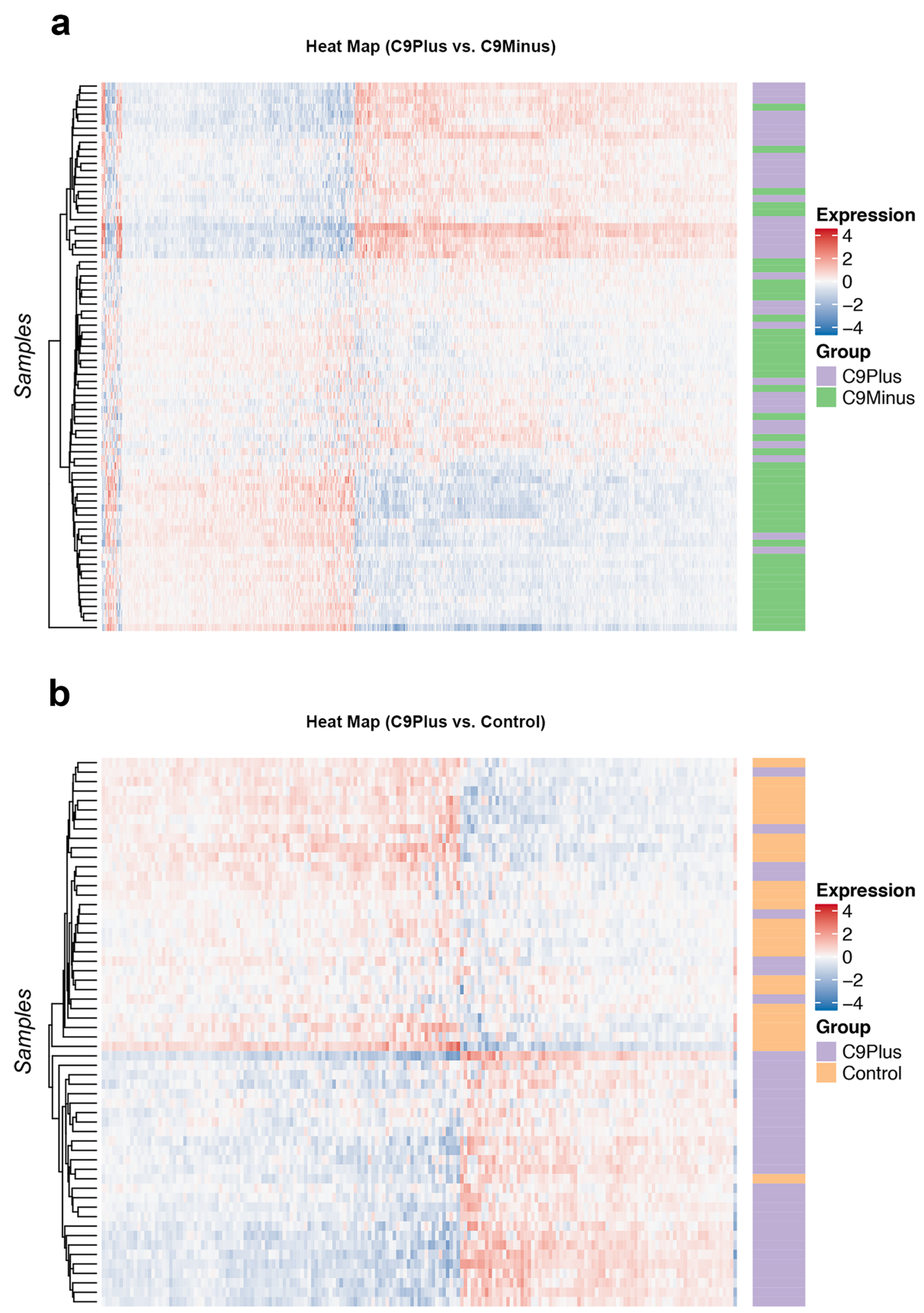

Fig. 2 a When comparing patients with a C9orf72 repeat expansion to those without this expansion (C9Plus vs. C9Minus), a heat map is displayed. b A heat map is shown when comparing expansion carriers to control subjects (C9Plus vs. Control). In these heat maps, high expression levels are shown in red and low levels in blue. Both heat maps indicate that most expansion carriers cluster together (purple). Of note, for visualization purposes, only the top differentially expressed genes are displayed (false discovery rate $[\mathrm{FDR}]<0.001$ )

5-carboxylate reductase 2 (PYCR2), $5^{\prime}$-nucleotidase domain containing 1 (NT5DC1), S100 calcium binding protein B $(S 100 B)$, and dynactin subunit 6 (DCTN6).

Of note, without adjustment for cell-type-specific markers, the strongest relationships were no longer observed for the disease group, but for our surrogate markers (Additional file 1: Figure S1). As an example, neurons were highly correlated with the turquoise module, when comparing C9orf72 expansion carriers to patients without this expansion (correlation: 0.82; Additional file 1: Figure S1a) or to control subjects (correlation: 0.83; Additional file 1: Figure S1b). Enrichment analysis confirmed that the turquoise module was enriched for synaptic signaling (FDR $=1.30 \mathrm{E}-53$ and $\mathrm{FDR}=2.09 \mathrm{E}-44$, 
Table 3 Differential Expression (Specific Comparisons)

\begin{tabular}{|c|c|c|c|c|c|c|c|c|c|c|}
\hline \multirow[t]{2}{*}{ Order } & \multicolumn{5}{|c|}{ C9Plus vs. C9Minus } & \multicolumn{5}{|c|}{ C9Plus vs. Control } \\
\hline & Gene & Chr & $P$-value & FDR & FC & Gene & Chr & $P$-value & FDR & $\mathrm{FC}$ \\
\hline 1 & C9orf72 & chr9 & $4.35 \mathrm{E}-10$ & $6.04 \mathrm{E}-06$ & -1.75 & C9orf72 & chr9 & $4.48 \mathrm{E}-10$ & $1.08 \mathrm{E}-05$ & -1.69 \\
\hline 2 & FBLLI & chr5 & $5.01 \mathrm{E}-10$ & $6.04 \mathrm{E}-06$ & 1.88 & SMIM14 & chr4 & $3.16 \mathrm{E}-09$ & $3.80 \mathrm{E}-05$ & -1.34 \\
\hline 3 & VSTM2A-OT1 & chr7 & $1.51 \mathrm{E}-09$ & $9.64 \mathrm{E}-06$ & 1.96 & PYCR2 & chr1 & $5.96 \mathrm{E}-09$ & $3.99 \mathrm{E}-05$ & -1.73 \\
\hline 4 & KCNJ6 & chr21 & $1.88 \mathrm{E}-09$ & $9.64 \mathrm{E}-06$ & 1.92 & NT5DC1 & chr6 & $9.62 \mathrm{E}-09$ & 3.99E-05 & -1.57 \\
\hline 5 & $A B C E 1$ & chr4 & 2.06E-09 & $9.64 \mathrm{E}-06$ & -1.35 & GID4 & chr17 & $1.08 \mathrm{E}-08$ & $3.99 \mathrm{E}-05$ & 1.33 \\
\hline 6 & SMIM14 & chr4 & 2.40E-09 & $9.64 \mathrm{E}-06$ & -1.25 & GlT1 & chr17 & 1.10E-08 & 3.99E-05 & 1.63 \\
\hline 7 & $\mathrm{HRH} 3$ & chr20 & 4.12E-09 & $1.40 \mathrm{E}-05$ & 1.99 & SCN3A & chr2 & $1.16 \mathrm{E}-08$ & $3.99 \mathrm{E}-05$ & 1.94 \\
\hline 8 & SH3GL1P3 & chr17 & 4.65E-09 & $1.40 \mathrm{E}-05$ & 1.80 & $S 100 B$ & chr21 & $1.38 \mathrm{E}-08$ & 4.17E-05 & -1.94 \\
\hline 9 & CTD-2126E3.4 & chr19 & 5.61E-09 & 1.50E-05 & 2.11 & CMTM5 & chr14 & $1.71 \mathrm{E}-08$ & 4.57E-05 & -2.49 \\
\hline 10 & LRFN4 & chr11 & 7.15E-09 & $1.72 \mathrm{E}-05$ & 1.69 & RP11-196E1.3 & chr11 & $1.96 \mathrm{E}-08$ & $4.72 \mathrm{E}-05$ & -2.90 \\
\hline 11 & RP11-147L13.13 & chr17 & $1.02 \mathrm{E}-08$ & 2.24E-05 & 1.80 & DCTN6 & chr8 & $3.88 \mathrm{E}-08$ & $6.82 \mathrm{E}-05$ & -1.33 \\
\hline 12 & $C A 7$ & chr16 & $1.14 \mathrm{E}-08$ & $2.28 \mathrm{E}-05$ & 1.69 & SUCLG2 & chr3 & 4.11E-08 & $6.82 \mathrm{E}-05$ & -1.72 \\
\hline 13 & MXD4 & chr4 & 1.36E-08 & 2.47E-05 & 1.35 & MARCKSLI & chr1 & 4.16E-08 & $6.82 \mathrm{E}-05$ & -1.57 \\
\hline 14 & STX12 & chr1 & $1.43 \mathrm{E}-08$ & $2.47 \mathrm{E}-05$ & -1.23 & KCNG1 & chr20 & 4.17E-08 & $6.82 \mathrm{E}-05$ & 2.88 \\
\hline 15 & ATG4D & chr19 & 1.73E-08 & $2.78 \mathrm{E}-05$ & 1.30 & $B A G 2$ & chr6 & 4.25E-08 & $6.82 \mathrm{E}-05$ & 1.47 \\
\hline 16 & DUSP14 & chr17 & $1.84 \mathrm{E}-08$ & $2.78 \mathrm{E}-05$ & 1.34 & RAF1 & chr3 & 5.09E-08 & 7.67E-05 & 1.23 \\
\hline 17 & CHMP2B & chr3 & 1.97E-08 & $2.78 \mathrm{E}-05$ & -1.32 & TESK1 & chr9 & 5.57E-08 & 7.81E-05 & 1.33 \\
\hline 18 & LPHN1 & chr19 & $2.08 \mathrm{E}-08$ & $2.78 \mathrm{E}-05$ & 1.43 & $B D N F-A S$ & chr11 & $6.20 \mathrm{E}-08$ & 7.81E-05 & -1.82 \\
\hline 19 & ST8SIA5 & chr18 & $2.31 \mathrm{E}-08$ & 2.93E-05 & 1.52 & CTC-273B12.10 & chr19 & $6.57 \mathrm{E}-08$ & 7.81E-05 & 2.69 \\
\hline 20 & NFATC2IP & chr16 & $2.55 \mathrm{E}-08$ & 3.07E-05 & -1.29 & CYP4F11 & chr19 & 6.61E-08 & 7.81E-05 & -3.25 \\
\hline
\end{tabular}

Differentially expressed genes are shown when comparing patients with an expanded C9orf72 repeat to those without this repeat (C9Plus vs. C9Minus) or to control subjects (C9Plus vs. Control). For each gene, the chromosome (Chr), $p$-value, false discovery rate (FDR), and fold-change (FC) are displayed. Of note, this table has been generated after adjustment for cell-type-specific markers

respectively). Similarly, microglia were strongly correlated with the grey60 module, demonstrating a correlation of 0.87 for both comparisons, while being enriched for the immune response $(\mathrm{FDR}=8.23 \mathrm{E}-62$ and $\mathrm{FDR}=1.51 \mathrm{E}-63$, respectively). The importance of our adjustment for celltype-specific markers was further substantiated by a cluster dendrogram (Additional file 1: Figure S2); branches in this dendrogram correspond to the modules we identified. After adjustment for cellular composition (Additional file 1: Figure S2a), the turquoise module was relatively small and seemed more closely related to the disease group than to our neuronal marker. Without this adjustment, however, the turquoise module was much larger and resembled the pattern of our neuronal marker (Additional file 1: Figure S2b). Importantly, without adjustment for surrogate markers, the green module involved in vesicular transport and the yellow module that contains C9orf72 still correlated with the disease group (Additional file 1: Figure S1 and S3), but findings were less prominent than those obtained after adjustment.

\section{Machine learning uncovers clinico-pathological associations}

We then performed an exploratory analysis aiming at the discovery of clinico-pathological associations, when restricting our cohort to FTLD and FTLD/MND patients

Table 4 Enrichment Analysis (Overlapping Genes)

\begin{tabular}{lllll}
\hline Source & Order & Process/Pathway & $P$-value & FDR \\
\hline KEGG & 1 & KEGG_ENDOCYTOSIS & 4.40 E-05 & 0.02 \\
& 2 & KEGG_CITRATE_CYCLE_TCA_CYCLE & 0.004 & 0.38 \\
GO-BP & 1 & GO_ORGANIC_ACID_METABOLIC_PROCESS & $7.91 \mathrm{E}-05$ & 0.27 \\
& 2 & GO_TRICARBOXYLIC_ACID_METABOLIC_PROCESS & 1.40 E-04 & 0.27 \\
\hline
\end{tabular}

Results of enrichment analyses are shown for overlapping genes. For each process or pathway, the $p$-value and false discovery rate (FDR) are included. Enrichment analyses were performed using Kyoto Encyclopedia of Genes and Genomes (KEGG) and Gene Ontology - Biological Processes (GO-BP). Of note, this table has been generated after adjustment for cell-type-specific markers 
a

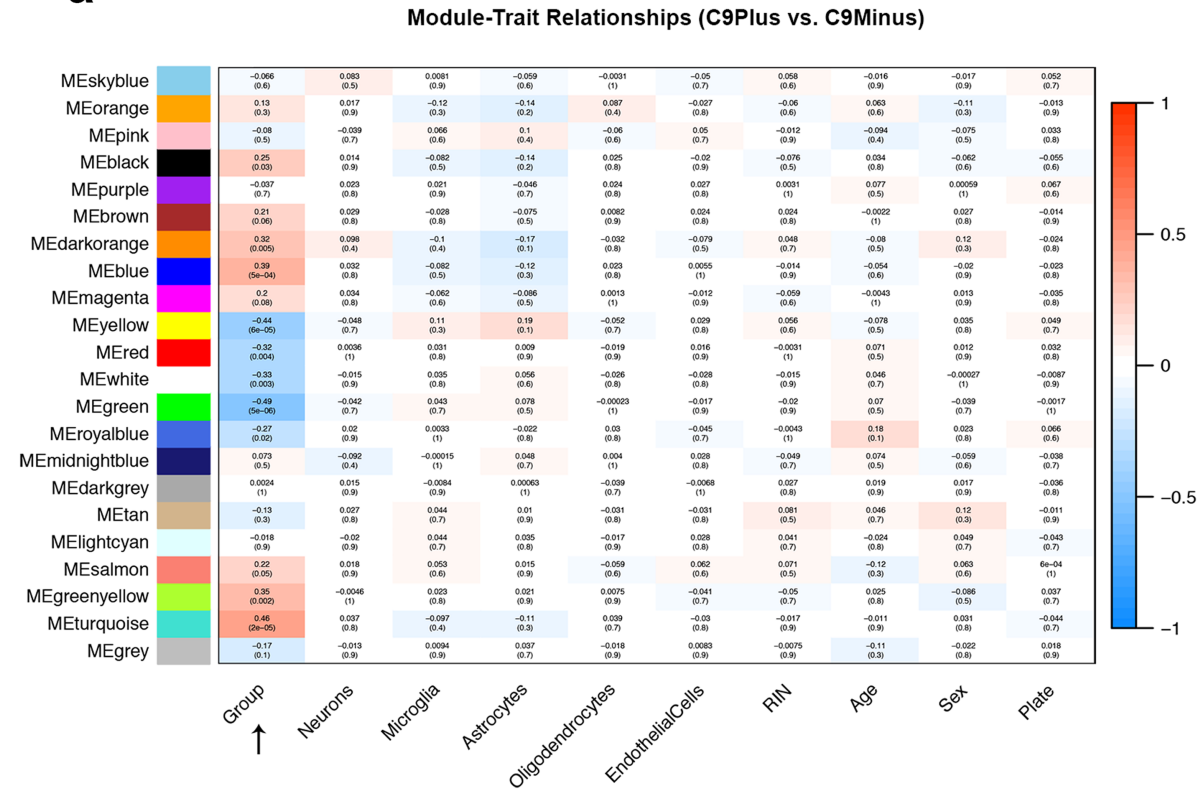

b

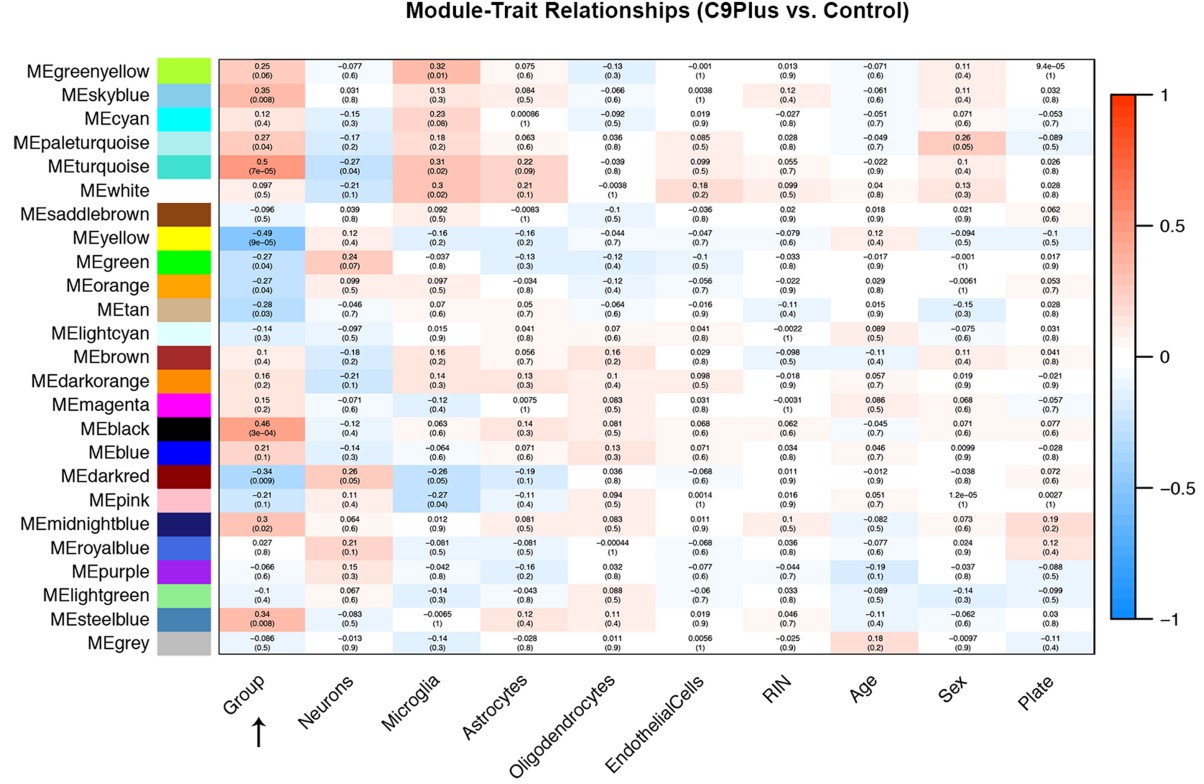

Fig. 3 a Module-trait relationships are presented for patients with an expanded C9orf72 repeat and patients without this repeat (C9Plus vs. C9Minus). b For patients with an expansion and control subjects (C9Plus vs. Control), module-trait relationships are plotted. These plots are generated with weighted gene co-expression network analysis (WGCNA) to find groups of genes that go up (red) or down (blue) together. A unique color has been assigned to each of these groups, also called a module. Correlations and $p$-values are shown for variables of interest, including disease group (C9Plus, C9Minus, and/or Control; arrow), neurons, microglia, astrocytes, oligodendrocytes, endothelial cells, RNA integrity number (RIN), age at death, sex, and plate. The strongest correlations (brightest colors) are observed for the disease group. Notably, both moduletrait relationship plots are based on residuals obtained after adjustment for cell-type-specific markers

harboring an expanded C9orf72 repeat $(n=34)$. Three types of models were used with residuals adjusted for cell-type-specific markers as input: linear regression models, logistic regression models, and Cox proportional hazard regression models. Our single-gene analysis did not reveal individual genes that remained significant after adjustment for multiple testing (not shown). Nonetheless, when analyzing all nominally significant genes, machine learning did point to interesting candidates, which were consistently associated with a given outcome 
Table 5 Enrichment Analysis (C9Plus vs. C9Minus)

\begin{tabular}{|c|c|c|c|c|c|c|}
\hline Module & Dir & Source & Order & Process/Pathway & $P$-value & FDR \\
\hline \multirow[t]{4}{*}{ MEblack } & \multirow[t]{4}{*}{ Up } & \multirow[t]{2}{*}{ KEGG } & 1 & KEGG_ANTIGEN_PROCESSING_AND_PRESENTATION & $3.31 \mathrm{E}-06$ & 4.34E-04 \\
\hline & & & 2 & KEGG_SPLICEOSOME & $3.80 \mathrm{E}-04$ & 0.02 \\
\hline & & \multirow[t]{2}{*}{ GO-BP } & 1 & GO_PROTEIN_FOLDING & $4.24 \mathrm{E}-29$ & $8.97 \mathrm{E}-26$ \\
\hline & & & 2 & GO_RESPONSE_TO_TOPOLOGICALLY_INCORRECT_PROTEIN & $6.40 \mathrm{E}-19$ & 7.55E-16 \\
\hline \multirow[t]{4}{*}{ MEdarkorange } & \multirow[t]{4}{*}{ Up } & \multirow[t]{2}{*}{ KEGG } & 1 & KEGG_ECM_RECEPTOR_INTERACTION & 0.04 & 0.67 \\
\hline & & & 2 & KEGG_FOCAL_ADHESION & 0.10 & 1.00 \\
\hline & & \multirow[t]{2}{*}{ GO-BP } & 1 & GO_PROTEIN_HETEROOLIGOMERIZATION & 0.001 & 0.06 \\
\hline & & & 2 & GO_REGULATION_OF_CARBOHYDRATE_METABOLIC_PROCESS & 0.003 & 0.14 \\
\hline \multirow[t]{4}{*}{ MEblue } & \multirow[t]{4}{*}{ Up } & \multirow[t]{2}{*}{ KEGG } & 1 & KEGG_SPLICEOSOME & 0.002 & 0.09 \\
\hline & & & 2 & KEGG_RNA_DEGRADATION & 0.04 & 0.68 \\
\hline & & \multirow[t]{2}{*}{ GO-BP } & 1 & GO_RNA_SPLICING & 7.85E-12 & 4.57E-09 \\
\hline & & & 2 & GO_RNA_PROCESSING & $9.98 \mathrm{E}-12$ & $5.56 \mathrm{E}-09$ \\
\hline \multirow[t]{4}{*}{ MEyellow } & \multirow[t]{4}{*}{ Down } & \multirow[t]{2}{*}{ KEGG } & 1 & KEGG_PEROXISOME & $4.48 \mathrm{E}-06$ & $5.00 \mathrm{E}-04$ \\
\hline & & & 2 & KEGG_DNA_REPLICATION & 3.65E-04 & 0.02 \\
\hline & & \multirow[t]{2}{*}{ GO-BP } & 1 & GO_SMALL_MOLECULE_METABOLIC_PROCESS & 4.33E-09 & $1.58 \mathrm{E}-06$ \\
\hline & & & 2 & GO_CELLULAR_AMINO_ACID_METABOLIC_PROCESS & $3.34 \mathrm{E}-08$ & $1.01 \mathrm{E}-05$ \\
\hline \multirow[t]{4}{*}{ MEred } & \multirow[t]{4}{*}{ Down } & \multirow[t]{2}{*}{ KEGG } & 1 & KEGG_ECM_RECEPTOR_INTERACTION & $1.52 \mathrm{E}-07$ & $3.38 \mathrm{E}-05$ \\
\hline & & & 2 & KEGG_FOCAL_ADHESION & 3.67E-06 & 4.34E-04 \\
\hline & & \multirow[t]{2}{*}{ GO-BP } & 1 & GO_EXTRACELLULAR_STRUCTURE_ORGANIZATION & $1.56 \mathrm{E}-16$ & $1.45 \mathrm{E}-13$ \\
\hline & & & 2 & GO_TISSUE_DEVELOPMENT & $2.61 \mathrm{E}-12$ & $1.54 \mathrm{E}-09$ \\
\hline \multirow[t]{4}{*}{ MEwhite } & \multirow[t]{4}{*}{ Down } & \multirow[t]{2}{*}{ KEGG } & 1 & KEGG_RIBOSOME & 1.49E-05 & 0.001 \\
\hline & & & 2 & KEGG_PROTEIN_EXPORT & 0.01 & 0.32 \\
\hline & & GO-BP & 1 & GO_PEPTIDE_METABOLIC_PROCESS & $1.57 \mathrm{E}-07$ & 4.08E-05 \\
\hline & & & 2 & GO_CELLULAR_AMIDE_METABOLIC_PROCESS & $6.22 \mathrm{E}-07$ & 1.34E-04 \\
\hline MEgreen & Down & KEGG & 1 & KEGG_PARKINSONS_DISEASE & $1.74 \mathrm{E}-08$ & 5.23E-06 \\
\hline & & & 2 & KEGG_HUNTINGTONS_DISEASE & $1.50 \mathrm{E}-06$ & 2.17E-04 \\
\hline & & GO-BP & 1 & GO_GOLGI_VESICLE_TRANSPORT & 3.53E-09 & $1.33 \mathrm{E}-06$ \\
\hline & & & 2 & GO_SMALL_MOLECULE_METABOLIC_PROCESS & $5.83 \mathrm{E}-09$ & 2.05E-06 \\
\hline MEroyalblue & Down & KEGG & 1 & KEGG_FOCAL_ADHESION & $1.56 \mathrm{E}-07$ & $3.38 \mathrm{E}-05$ \\
\hline & & & 2 & KEGG_COMPLEMENT_AND_COAGULATION_CASCADES & 2.70E-07 & 5.03E-05 \\
\hline & & GO-BP & 1 & GO_VASCULATURE_DEVELOPMENT & $2.11 \mathrm{E}-17$ & 2.18E-14 \\
\hline & & & 2 & GO_BLOOD_VESSEL_MORPHOGENESIS & $4.64 \mathrm{E}-17$ & 4.65E-14 \\
\hline MEsalmon & Up & KEGG & 1 & KEGG_PROTEASOME & $3.80 \mathrm{E}-06$ & 4.36E-04 \\
\hline & & & 2 & KEGG_ALZHEIMERS_DISEASE & $2.59 \mathrm{E}-04$ & 0.02 \\
\hline & & GO-BP & 1 & GO_MITOCHONDRION_ORGANIZATION & $1.02 \mathrm{E}-11$ & $5.64 \mathrm{E}-09$ \\
\hline & & & 2 & GO_CELLULAR_MACROMOLECULAR_COMPLEX_ASSEMBLY & $2.34 \mathrm{E}-08$ & 7.38E-06 \\
\hline MEgreenyellow & Up & KEGG & 1 & KEGG_TAURINE_AND_HYPOTAURINE_METABOLISM & $1.26 \mathrm{E}-04$ & 0.009 \\
\hline & & & 2 & KEGG_BETA_ALANINE_METABOLISM & $9.26 \mathrm{E}-04$ & 0.04 \\
\hline & & GO-BP & 1 & GO_CEREBRAL_CORTEX_GABAERGIC_INTERNEURON_DIFFERENTIATION & $3.80 \mathrm{E}-09$ & $1.41 \mathrm{E}-06$ \\
\hline & & & 2 & GO_GABAERGIC_NEURON_DIFFERENTIATION & $8.92 \mathrm{E}-09$ & $3.02 \mathrm{E}-06$ \\
\hline MEturquoise & Up & KEGG & 1 & KEGG_NEUROACTIVE_LIGAND_RECEPTOR_INTERACTION & $6.26 \mathrm{E}-13$ & $2.72 \mathrm{E}-10$ \\
\hline & & & 2 & KEGG_CALCIUM_SIGNALING_PATHWAY & 3.22E-09 & $1.14 \mathrm{E}-06$ \\
\hline & & GO-BP & 1 & GO_SYNAPTIC_SIGNALING & $2.36 \mathrm{E}-50$ & $6.29 \mathrm{E}-47$ \\
\hline & & & 2 & GO_MODULATION_OF_SYNAPTIC_TRANSMISSION & $6.15 \mathrm{E}-40$ & 1.47E-36 \\
\hline
\end{tabular}

For significant modules, results of the enrichment analysis are specified when comparing C9orf72 expansion carriers to patients without this expansion (C9Plus vs. C9Minus). Each time, the direction of the change (Dir), process or pathway, $p$-value, and false discovery rate (FDR) are incorporated. Kyoto Encyclopedia of Genes and Genomes (KEGG) and Gene Ontology - Biological Processes (GO-BP) were used. Of note, this table has been generated after adjustment for cell-type-specific markers 


\section{a}

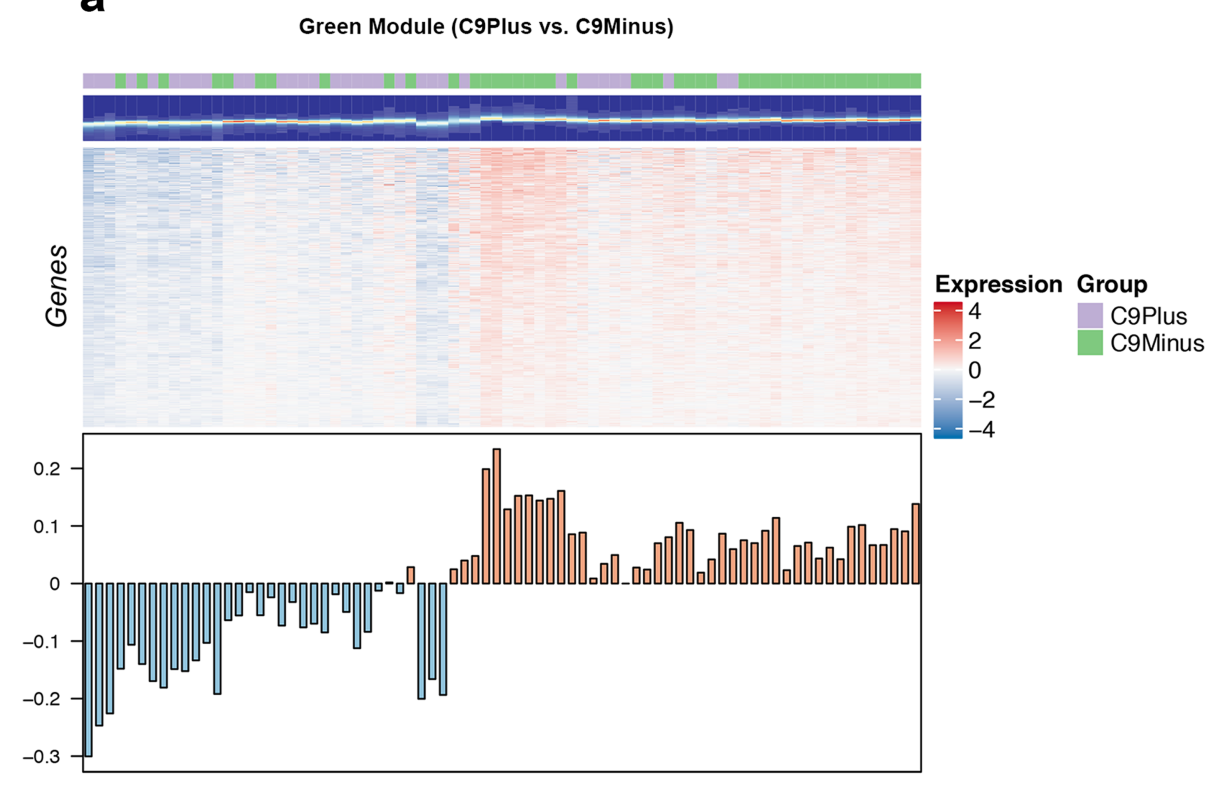

b

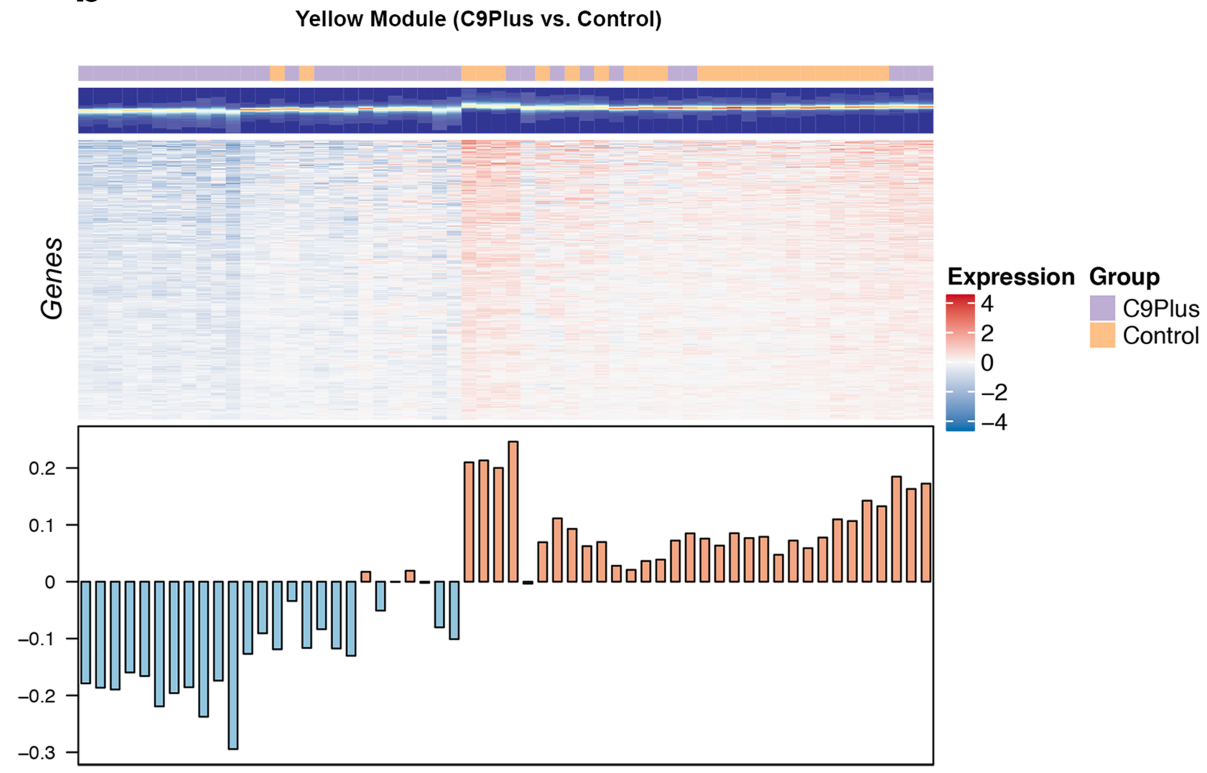

Fig. 4 a One specific group of genes is visualized in a heat map: the green module. $\mathbf{b}$ A heat map is displayed for the yellow module. High expression levels are shown in red and low levels in blue. Below every heat map, the first principal component of a given module (module eigengene) is displayed for each sample. Most C9orf72 expansion carriers (C9Plus) appear to have relatively low levels as compared to patients without this expansion (C9Minus) or to control subjects (Control)

using multiple methods and which were biologically relevant.

The most parsimonious models generated by LASSO regression contained up to 13 genes, depending on the variable studied (Table 7). When focusing on age at onset as response variable, for instance, only one gene was found: VEGFA (Fig. 6a). Importantly, this gene was the 10th gene based on our random forest analysis (Fig. 7a), and additionally, it was the 6th gene in our single-gene analysis $(P=$ 9.17E-05). One of the four genes selected by LASSO regression that seemed associated with $C 9$ orf 72 expansion size was CDKL1 (Fig. 6b). This gene was listed as the 19th gene in the random forest analysis (Fig. 7b) and the top gene in the single-gene analysis $(P=5.28 \mathrm{E}-05)$. Another interesting gene identified by 


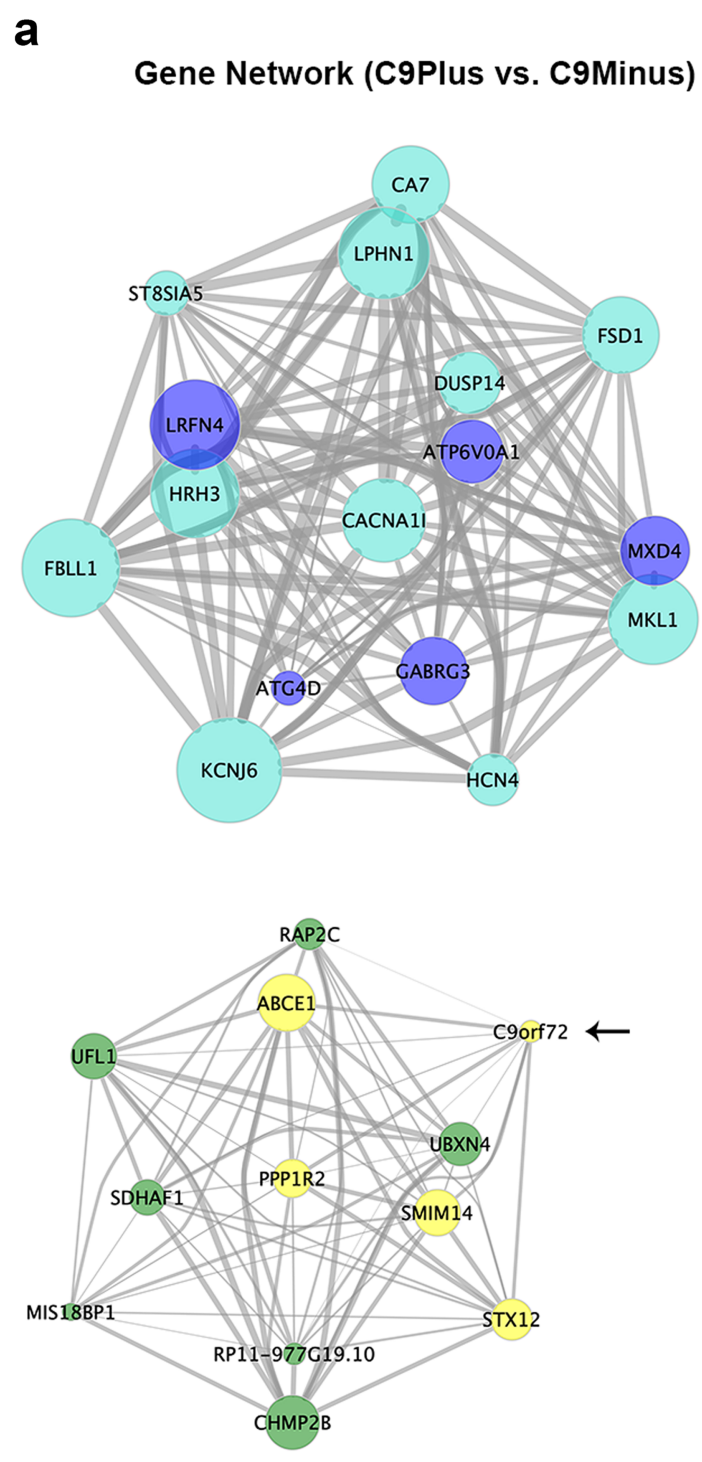

\section{b}

\section{Gene Network (C9Plus vs. Control)}
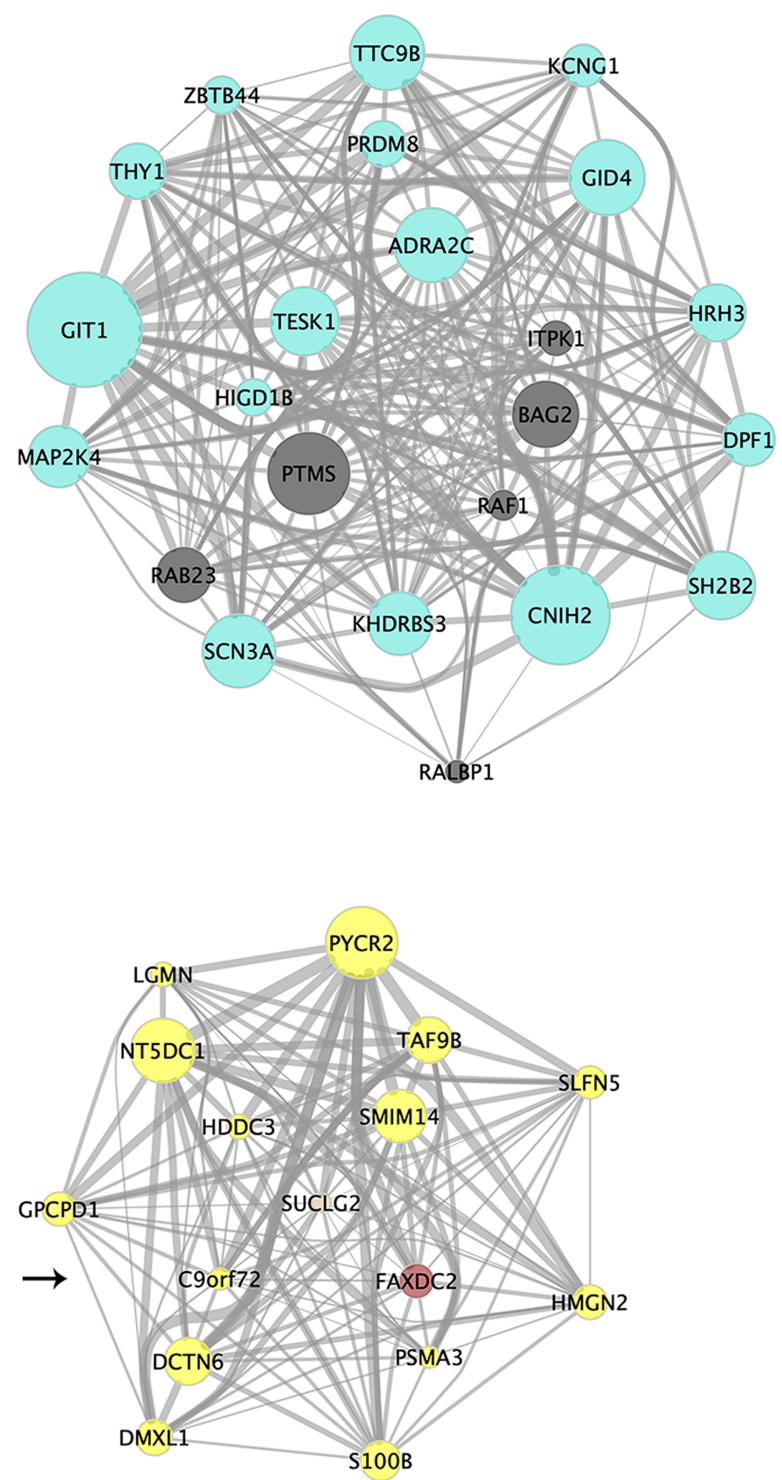

Fig. 5 a For patients harboring a C9orf72 repeat expansion and those without this expansion (C9Plus vs. C9Minus; module membership > 0.6 and significance < 1.0E-06), a gene network is displayed. b A gene network is visualized when examining expansion carriers and controls (C9Plus vs. Control; module membership $>0.6$ and significance < 2.5E-05). In these network plots, the connectivity of each gene is represented by the size of its node, the module to which it has been assigned by its color, and the strength of the correlation by the thickness of its edges; the C9orf72 gene is denoted by an arrow. Of note, the plots in this figure have been generated after adjustment for cell-type-specific markers

LASSO regression was $E E F 2 K$, which appeared to be associated with the level of poly(GP) proteins (Fig. $6 \mathrm{c})$. This gene was also the 3rd most important variable according to a random forest algorithm (Fig. 7c) and the 6th gene according to the single-gene analysis $(P=9.69 \mathrm{E}-04)$. Without adjustment for surrogate markers, similar trends were observed for VEGFA $(P=9.47 \mathrm{E}-04), \quad C D K L 1 \quad(P=0.01)$, and EEF2K $(P=$ 0.002; Additional file 1: Figure S4a-c).
In the survival after onset model, LASSO regression identified two genes, one of which was a gene called SGSM3 that was the top hit of our single-gene analysis $(P=1.31 \mathrm{E}-05 ;$ Table 7$)$. In patients belonging to the bottom $50 \%$ of SGSM3 expression levels, the median survival after onset was 4.8 years (IQR: 3.0-6.8) versus 8.6 years in the top $50 \%$ (IQR: 7.5-12.1; Fig. 6d). This difference resulted in an HR of 0.10 (95\% CI: 0.04-0.28). We were able to confirm these findings when analyzing 
Table 6 Enrichment Analysis (C9Plus vs. Control)

\begin{tabular}{|c|c|c|c|c|c|c|}
\hline Module & Dir & Source & Order & Process/Pathway & $P$-value & FDR \\
\hline \multirow[t]{4}{*}{ MEskyblue } & \multirow[t]{4}{*}{ Up } & \multirow[t]{2}{*}{ KEGG } & 1 & KEGG_MELANOGENESIS & 4.24E-04 & 0.04 \\
\hline & & & 2 & KEGG_NOTCH_SIGNALING_PATHWAY & 7.46E-04 & 0.06 \\
\hline & & \multirow[t]{2}{*}{ GO-BP } & 1 & GO_POSITIVE_REGULATION_OF_BIOSYNTHETIC_PROCESS & 5.27E-05 & 0.008 \\
\hline & & & 2 & $\begin{array}{l}\text { GO_REGULATION_OF_INTRACELLULAR_STEROID_HORMONE } \\
\text { RECEPTOR_SIGNALING_PATHWAY }\end{array}$ & $5.66 \mathrm{E}-05$ & 0.009 \\
\hline \multirow[t]{4}{*}{ MEpaleturquoise } & \multirow[t]{4}{*}{ Up } & \multirow[t]{2}{*}{ KEGG } & 1 & KEGG_NEUROACTIVE_LIGAND_RECEPTOR_INTERACTION & $1.09 \mathrm{E}-04$ & 0.01 \\
\hline & & & 2 & KEGG_TAURINE_AND_HYPOTAURINE_METABOLISM & 1.80E-04 & 0.02 \\
\hline & & \multirow[t]{2}{*}{ GO-BP } & 1 & GO_CEREBRAL_CORTEX_GABAERGIC_INTERNEURON_DIFFERENTIATION & 7.95E-09 & 3.97E-06 \\
\hline & & & 2 & GO_GABAERGIC_NEURON_DIFFERENTIATION & 1.87E-08 & $8.41 \mathrm{E}-06$ \\
\hline \multirow[t]{4}{*}{ MEturquoise } & \multirow[t]{4}{*}{ Up } & \multirow[t]{2}{*}{ KEGG } & 1 & KEGG_NEUROACTIVE_LIGAND_RECEPTOR_INTERACTION & 4.56E-15 & $2.26 \mathrm{E}-12$ \\
\hline & & & 2 & KEGG_CALCIUM_SIGNALING_PATHWAY & 2.44E-06 & $6.06 \mathrm{E}-04$ \\
\hline & & \multirow[t]{2}{*}{ GO-BP } & 1 & GO_SYNAPTIC_SIGNALING & $3.79 E-33$ & $1.06 \mathrm{E}-29$ \\
\hline & & & 2 & GO_CELL_CELL_SIGNALING & $3.59 \mathrm{E}-23$ & $8.31 \mathrm{E}-20$ \\
\hline \multirow[t]{4}{*}{ MEyellow } & \multirow[t]{4}{*}{ Down } & \multirow[t]{2}{*}{ KEGG } & 1 & KEGG_PEROXISOME & $5.76 \mathrm{E}-07$ & $1.71 \mathrm{E}-04$ \\
\hline & & & 2 & KEGG_VALINE_LEUCINE_AND_ISOLEUCINE_DEGRADATION & $1.60 \mathrm{E}-05$ & 0.003 \\
\hline & & \multirow[t]{2}{*}{ GO-BP } & 1 & GO_SMALL_MOLECULE_METABOLIC_PROCESS & $1.44 \mathrm{E}-16$ & $2.10 \mathrm{E}-13$ \\
\hline & & & 2 & GO_ORGANIC_ACID_CATABOLIC_PROCESS & $1.23 \mathrm{E}-14$ & $1.39 \mathrm{E}-11$ \\
\hline \multirow[t]{4}{*}{ MEgreen } & \multirow[t]{4}{*}{ Down } & \multirow[t]{2}{*}{ KEGG } & 1 & KEGG_CITRATE_CYCLE_TCA_CYCLE & $6.15 \mathrm{E}-05$ & 0.009 \\
\hline & & & 2 & KEGG_PYRUVATE_METABOLISM & 0.002 & 0.11 \\
\hline & & \multirow[t]{2}{*}{ GO-BP } & 1 & GO_ORGANIC_ACID_METABOLIC_PROCESS & $6.24 \mathrm{E}-08$ & $2.49 \mathrm{E}-05$ \\
\hline & & & 2 & GO_GOLGI_VESICLE_TRANSPORT & 7.76E-08 & $2.98 \mathrm{E}-05$ \\
\hline \multirow[t]{4}{*}{ MEorange } & \multirow[t]{4}{*}{ Down } & \multirow[t]{2}{*}{ KEGG } & 1 & KEGG_PARKINSONS_DISEASE & 4.93E-34 & $1.10 \mathrm{E}-30$ \\
\hline & & & 2 & KEGG_OXIDATIVE_PHOSPHORYLATION & $3.39 E-32$ & $5.04 \mathrm{E}-29$ \\
\hline & & GO-BP & 1 & GO_NUCLEOSIDE_TRIPHOSPHATE_METABOLIC_PROCESS & $5.35 \mathrm{E}-29$ & $1.46 \mathrm{E}-25$ \\
\hline & & & 2 & GO_OXIDATIVE_PHOSPHORYLATION & $1.74 \mathrm{E}-28$ & 4.63E-25 \\
\hline MEtan & Down & KEGG & 1 & KEGG_GLYCINE_SERINE_AND_THREONINE_METABOLISM & $5.51 \mathrm{E}-07$ & $1.71 \mathrm{E}-04$ \\
\hline & & & 2 & KEGG_FATTY_ACID_METABOLISM & $6.55 \mathrm{E}-07$ & $1.83 \mathrm{E}-04$ \\
\hline & & GO-BP & 1 & GO_OXIDATION_REDUCTION_PROCESS & 8.53E-18 & $1.40 \mathrm{E}-14$ \\
\hline & & & 2 & GO_BIOLOGICAL_ADHESION & $1.39 \mathrm{E}-15$ & $1.93 \mathrm{E}-12$ \\
\hline MEblack' & Up & KEGG & 1 & KEGG_BASAL_TRANSCRIPTION_FACTORS & 0.002 & 0.11 \\
\hline & & & 2 & KEGG_SPLICEOSOME & 0.02 & 0.48 \\
\hline & & GO-BP & 1 & GO_NEGATIVE_REGULATION_OF_NITROGEN_COMPOUND_METABOLIC_PROCESS & $6.04 \mathrm{E}-09$ & $3.11 \mathrm{E}-06$ \\
\hline & & & 2 & GO_CHROMATIN_MODIFICATION & 4.90E-08 & $2.00 \mathrm{E}-05$ \\
\hline MEdarkred & Down & KEGG & 1 & KEGG_LYSOSOME & 0.004 & 0.18 \\
\hline & & & 2 & KEGG_STEROID_BIOSYNTHESIS & 0.005 & 0.19 \\
\hline & & GO-BP & 1 & GO_ENSHEATHMENT_OF_NEURONS & 4.31E-05 & 0.007 \\
\hline & & & 2 & GO_NEGATIVE_REGULATION_OF_CELLULAR_COMPONENT_ORGANIZATION & $5.22 \mathrm{E}-05$ & 0.008 \\
\hline MEmidnightblue & Up & KEGG & 1 & KEGG_ANTIGEN_PROCESSING_AND_PRESENTATION & $5.26 \mathrm{E}-05$ & 0.008 \\
\hline & & & 2 & KEGG_SYSTEMIC_LUPUS_ERYTHEMATOSUS & 0.001 & 0.09 \\
\hline & & GO-BP & 1 & GO_PROTEIN_FOLDING & $2.29 \mathrm{E}-21$ & $4.69 \mathrm{E}-18$ \\
\hline & & & 2 & GO_PROTEIN_REFOLDING & 9.27E-12 & $8.16 \mathrm{E}-09$ \\
\hline MEsteelblue & Up & KEGG & 1 & KEGG_CYTOKINE_CYTOKINE_RECEPTOR_INTERACTION & 0.001 & 0.09 \\
\hline & & & 2 & KEGG_APOPTOSIS & 0.002 & 0.11 \\
\hline & & GO-BP & 1 & GO_INFLAMMATORY_RESPONSE & 7.44E-06 & 0.002 \\
\hline & & & 2 & GO_CELL_DEATH & $1.23 \mathrm{E}-05$ & 0.002 \\
\hline
\end{tabular}

For significant modules, results of the enrichment analysis are specified when comparing C9orf72 expansion carriers to control subjects (C9Plus vs. Control). Each time, the direction of the change (Dir), process or pathway, p-value, and false discovery rate (FDR) are incorporated. Kyoto Encyclopedia of Genes and Genomes (KEGG) and Gene Ontology - Biological Processes (GO-BP) were used. Of note, this table has been generated after adjustment for cell-type-specific markers 
Table 7 LASSO Regression

\begin{tabular}{|c|c|c|c|c|c|c|c|}
\hline \multirow[t]{2}{*}{ Variable } & \multirow[t]{2}{*}{ Order } & \multicolumn{3}{|l|}{ LASSO } & \multicolumn{3}{|c|}{ Single-Gene } \\
\hline & & Gene & Chr & Coef1 & Coef2 & SE & $P$-value \\
\hline Age at Onset & 1 & VEGFA & chr6 & 0.14 & 7.36 & 1.61 & $9.17 \mathrm{E}-05$ \\
\hline \multirow[t]{13}{*}{ Age at Death } & 1 & AUTS2 & chr7 & 0.68 & 23.86 & 4.64 & $1.41 \mathrm{E}-05$ \\
\hline & 2 & TAF8 & chr6 & 7.00 & 28.34 & 6.58 & 1.55E-04 \\
\hline & 3 & NAGS & chr17 & 0.16 & 10.87 & 2.58 & $2.02 \mathrm{E}-04$ \\
\hline & 4 & RP1-130H16.18 & chr22 & 2.88 & 15.96 & 3.82 & 2.23E-04 \\
\hline & 5 & DOCK10 & chr2 & -1.31 & -13.55 & 3.26 & $2.38 \mathrm{E}-04$ \\
\hline & 6 & CLEC4E & chr12 & 0.56 & 4.53 & 1.09 & 2.43E-04 \\
\hline & 7 & $A P O B R$ & chr16 & 0.88 & 6.59 & 1.60 & 2.71E-04 \\
\hline & 8 & LYST & chr1 & -5.12 & -29.58 & 7.43 & 3.86E-04 \\
\hline & 9 & SLC39A11 & chr17 & 0.25 & 13.82 & 3.48 & 3.96E-04 \\
\hline & 10 & $R P 11-3 G 21.1$ & chr8 & 0.29 & 6.95 & 1.78 & 4.67E-04 \\
\hline & 11 & TIMM13 & chr19 & -0.38 & -22.98 & 6.48 & 0.001 \\
\hline & 12 & $V C X$ & $\operatorname{chrX}$ & 3.39 & 12.55 & 3.56 & 0.001 \\
\hline & 13 & SELPLG & chr12 & 0.05 & 6.55 & 2.08 & 0.004 \\
\hline \multirow[t]{4}{*}{ Expansion Size } & 1 & $C D K L 1$ & chr14 & 3.06 & 21.39 & 4.52 & $5.28 \mathrm{E}-05$ \\
\hline & 2 & FAM87A & chr8 & -0.50 & -9.21 & 2.24 & 2.94E-04 \\
\hline & 3 & $R P 11-481 J 2.3$ & chr16 & 0.64 & 7.52 & 2.14 & 0.001 \\
\hline & 4 & EFCAB14 & chr1 & -2.38 & -34.03 & 9.98 & 0.002 \\
\hline Sense RNA Foci & 1 & CATIP-AS1 & chr2 & -0.002 & -0.10 & 0.02 & $6.34 \mathrm{E}-05$ \\
\hline Antisense RNA Foci & 1 & CCDC127 & chr5 & 0.003 & 0.16 & 0.04 & 0.001 \\
\hline Poly(GP) & 1 & $E E F 2 K$ & chr16 & -10.11 & -495.81 & 133.94 & $9.69 \mathrm{E}-04$ \\
\hline \multirow[t]{3}{*}{ Methylation } & 1 & AC006946.16 & chr22 & -0.03 & -8.14 & 2.05 & 4.32E-04 \\
\hline & 2 & RP11-555 M1.3 & chr3 & 0.07 & 4.18 & 1.33 & 0.004 \\
\hline & 3 & $A 1 B G$ & chr19 & -0.009 & -8.69 & 2.78 & 0.004 \\
\hline \multirow[t]{6}{*}{ Disease Subgroup } & 1 & TMPPE & chr3 & 0.02 & 3.03 & 1.10 & 0.006 \\
\hline & 2 & EDEM2 & chr20 & 0.93 & 8.44 & 3.13 & 0.007 \\
\hline & 3 & CD37 & chr19 & -0.39 & -6.42 & 2.41 & 0.008 \\
\hline & 4 & $M Z T 2 B$ & chr2 & -1.15 & -22.86 & 8.75 & 0.009 \\
\hline & 5 & PLK5 & chr19 & -0.02 & -2.52 & 1.00 & 0.01 \\
\hline & 6 & NOTUM & chr17 & -0.32 & -4.87 & 2.30 & 0.03 \\
\hline Variable & Order & Gene & Chr & Coef1 & $\mathrm{HR}$ & $\mathrm{Cl}$ & $P$-value \\
\hline \multirow[t]{2}{*}{ Survival after Onset } & 1 & SGSM3 & chr22 & -0.02 & 0.10 & $0.04-0.28$ & $1.31 \mathrm{E}-05$ \\
\hline & 2 & EFNA2 & chr19 & -0.03 & 0.19 & $0.07-0.51$ & 0.001 \\
\hline
\end{tabular}

Genes selected using Least Absolute Shrinkage and Selection Operator (LASSO) regression are shown for variables of interest. The chromosome (Chr) and LASSO coefficient (Coef1) are included. Additionally, the results of the single-gene analysis are displayed for genes identified through LASSO regression, including the coefficient (Coef2), standard error (SE), hazard ratio (HR), 95\% confidence interval (CI), and/or $p$-value. Genes denoted by a bold font are displayed in Fig. 6 . Of note, this table has been generated after adjustment for cell-type-specific markers

expression levels based on rank, listing SGSM3 as the 3rd gene $(P=6.03 \mathrm{E}-04)$. Likewise, when treating expression levels as a continuous variable, SGSM3 was the 13th gene on the list $(P=0.001)$. Although much less profound, this trend with survival after onset was also observed without adjustment for cell-type-specific markers
( $P=0.02$; Additional file 1: Figure S4d). Together, our findings suggest that lower levels of SGSM3 might be associated with shortened survival after onset in C9orf72 expansion carriers. Notably, of our four genes of interest, SGSM3 was the only gene that was significantly differentially expressed between disease groups 


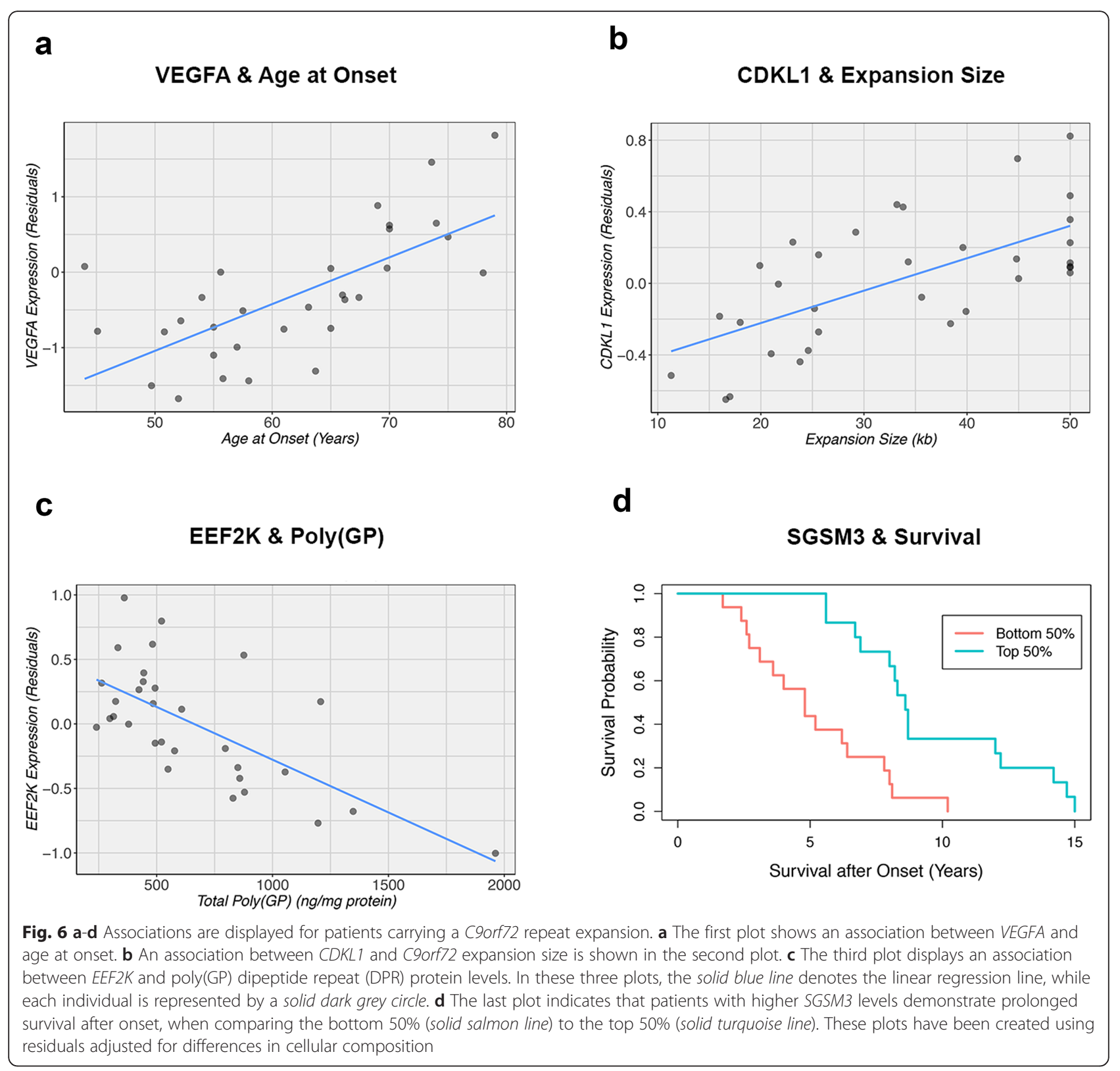

$(F D R=0.03)$, demonstrating elevated levels in patients carrying an expanded $C 9$ orf 72 repeat (Additional file 1: Figure S5).

We then used TaqMan expression assays for the four top candidate genes to validate the expression results from our RNA sequencing experiment in C9orf72 expansion carriers. When using residuals unadjusted for cellular composition, a significant correlation between our expression assays and RNA sequencing data was found for VEGFA ( $P=4.17 \mathrm{E}-05$, correlation: 0.68$), C D K L 1$ ( $P=0.003$, correlation: 0.55$), E E F 2 K(P=0.03$, correlation: 0.40$)$, and SGSM3 ( $P=0.03$, correlation: 0.40 ; Additional file 1 : Figure S6b, d, f, h). Similar correlations were obtained when using residuals adjusted for our five surrogate markers (Additional file 1: Figure S6a, c, e, g).

\section{Discussion}

In this study, we characterized the expression pattern of C9orf72-related diseases in an affected brain region: the frontal cortex. We examined FTLD and FTLD/MND patients with or without a C9orf72 repeat expansion as well as control subjects $(n=102)$. Differential expression analysis identified $C 9$ orf 72 as the top gene; it was approximately $50 \%$ reduced in C9orf 72 expansion carriers. Importantly, differentially expressed genes were enriched for endocytosis (FDR $=0.02)$. Without adjustment for 


\section{a}

\section{Gene Importance \& Age at Onset}

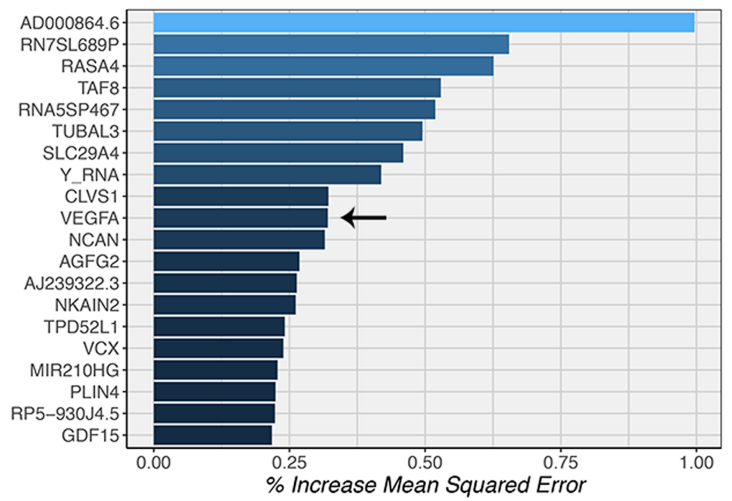

\section{b}

\section{Gene Importance \& Expansion Size}

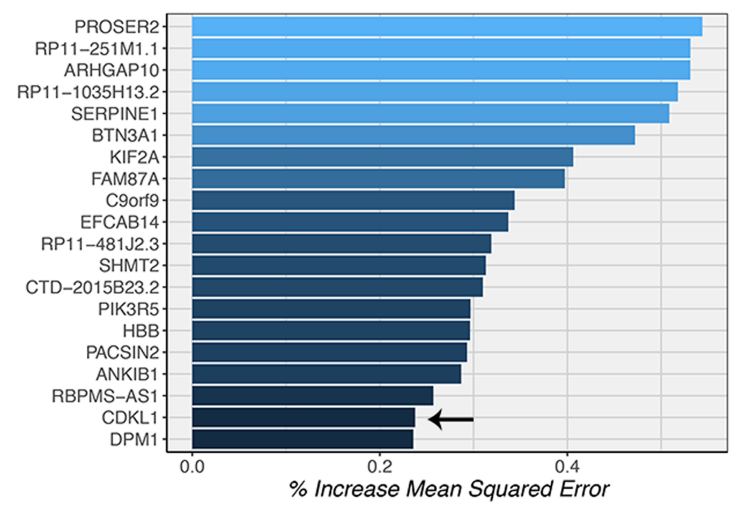

C

Gene Importance \& Poly(GP)

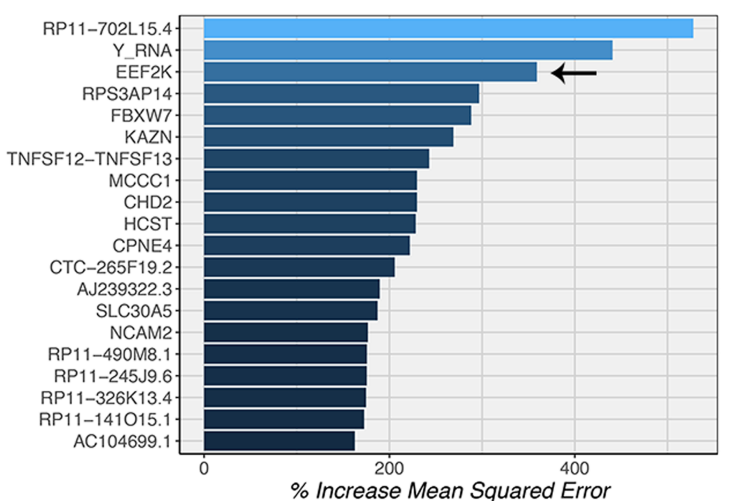

Fig. 7 a-c The importance of genes is visualized in three plots based on a random forest analysis. For continuous variables (age at onset, C9orf72 expansion size, and poly[GP] levels), the importance is defined as an increase in mean squared error. The blue gradient represents the importance of each gene, from very important (light) to less important (dark). Arrows point at genes of interest, namely VEGFA, CDKL1, and EEF2K (Table 7 and Fig. 6)

cell-type-specific markers, our co-expression analysis revealed modules influenced by neuronal loss (turquoise) and inflammation (grey60). Usage of surrogate markers resulted in the discovery of additional modules that correlated with the disease group, including modules enriched for protein folding, RNA processing, metabolic processes, and vesicle-mediated transport. The C9orf 72 gene itself was assigned to a module involved in metabolism (yellow) and clustered with genes belonging to a module that plays a role in vesicular transport (green). To identify potential disease modifiers, we then focused on the subset of individuals with an expanded repeat in C9orf72 ( $n=34)$. We used various analytical approaches, including LASSO regression and random forest, which pointed to promising candidates. In addition to VEGFA, for instance, we detected CDKL1, EEF2K, and SGSM3. Taken together, our RNA sequencing study uncovered that vital processes, such as vesicle transport, are affected by the presence of a repeat expansion in C9orf 72 . Furthermore, the modifiers identified in this study may represent biomarkers and/or therapeutic targets, which are in great demand.

Although the C9orf72 protein has been studied extensively since the discovery of a repeat expansion in the C9orf72 gene [14, 50], little is known about its function. It has been suggested that C9orf72 is a member of a superfamily called differentially expressed in normal and neoplasia (DENN) $[36,65]$, which contains GDP/GTP exchange factors (GEFs) that activate regulators of membrane trafficking known as Rab-GTPases. The C9orf72 protein has already been shown to co-localize with RabGTPases involved in endosomal transport [18]. Additionally, C9orf72 was found to form a complex with another DENN protein (SMCR8), serving as a GEF for specific 
Rab-GTPases [2, 53, 62, 64]. Furthermore, the C9orf72 protein appears to play a role in lysosomal biogenesis in addition to vesicle trafficking [56]. The presence of the C9orf72 repeat expansion seems to cause defects in vesicle trafficking and dysfunctional trans-Golgi network phenotypes, which can be reversed by overexpression of $C 9$ orf 72 or antisense oligonucleotides targeting the expanded repeat [3]. Interestingly, modulation of vesicle trafficking may even rescue neurodegeneration in induced motor neurons from C9orf72 expansion carriers [56].

Our study, in which we compared the expression pattern of C9orf72 expansion carriers to (disease) controls, uncovered $C 9$ orf 72 as the top hit of our differential expression analysis. This aligns with one of our previous studies where we detected reduced levels of $C 9$ orf 72 transcripts in expansion carriers and where we observed clinico-pathological associations with specific transcript variants [59]. It was reassuring to see that differentially expressed genes were enriched for endocytosis, especially given the potential role of the C9orf72 protein in vesicular transport. These findings were further substantiated by the fact that our co-expression analysis revealed a module that was enriched for Golgi vesicle transport as well as endoplasmic reticulum to Golgi vesicle-mediated transport, vacuolar transport, vesicle-mediated transport, and lysosomes. Our RNA sequencing study, therefore, provides additional evidence that the presence of a C9orf 72 repeat expansion might disrupt vesicle trafficking, a crucial process. Interestingly, we also discovered a promising modifier of survival after onset that is involved in vesicle transport: SGSM3. Our findings indicate that low expression levels of SGSM3 could be detrimental in C9orf72 expansion carriers, while high levels might have protective effects. The SGSM3 protein interacts with Ras-related protein Rab-8A [63], a small Rab-GTPase that is also regulated by the C9orf72-SMCR8 complex [53]. Consequently, one could postulate that higher levels of SGSM3 might counteract some of the harmful effects associated with an expanded repeat in C9orf72. In fact, a recent yeast screen demonstrated that $m s b 3$, the yeast ortholog of SGSM3, modifies the toxicity of one of the DPR proteins: poly(GR) [9]; other potential mechanisms seem worthy of exploration.

Another interesting candidate we identified, VEGFA, appeared to be associated with the age at which disease symptoms occur. Our findings suggest that higher expression levels of this gene are associated with a delayed age at onset ( $P=9.17 \mathrm{E}-05$, coefficient: 7.36$)$. While age at onset and age at death are strongly correlated, one could speculate that VEGFA levels might simply increase as an individual ages. Our single-gene analysis, however, revealed a stronger association with age at onset than with age at death $(P=0.003$, coefficient: 5.81$)$. The VEGFA protein belongs to the vascular endothelial growth factor
(VEGF) family and is thought to have neurotrophic effects $[28,29]$. Remarkably, reduced expression of Vegfa has been shown to cause an ALS-like phenotype in mice [45]. At the same time, treatment with Vegfa might protect motor neurons against ischemic death [32]. Additionally, genetic variants in VEGFA may render individuals more vulnerable to the development of ALS [31, 32]. Notably, neither an association with survival after onset $(P=0.26)$ nor a significant difference between disease subgroups (FTLD versus FTLD/ MND; $P=0.75$ ) was observed in our C9orf72 expansion carriers, but the association we detected with age at onset is in favor of a protective role for VEGFA.

In addition to SGSM3 and VEGFA, we also found associations with $C D K L 1$ and $E E F 2 K$. CDKL1 was associated with the size of $C 9$ orf 72 expansions: higher levels were observed in individuals with longer expansions. This gene is a member of the cyclin-dependent kinase family and appears to control the length of neuronal cilia [8]. At the moment, how CDKL1 possibly affects C9orf72 expansion size remains elusive. Expression levels of $E E F 2 K$ were associated with the amount of poly(GP); an increase in $E E F 2 K$ was seen in expansion carriers when poly(GP) levels decreased. It is a regulator of protein synthesis and synaptic plasticity that has already been studied in Alzheimer's disease and Parkinson's disease, where it may affect the toxicity of amyloid- $\beta$ and $\alpha$-synuclein [25-27]. Given the fact that it functions in protein synthesis and has previously been implicated in other neurodegenerative diseases, $E E F 2 K$ is an interesting candidate. Of note, for simplicity, we focused on four disease modifiers in this manuscript; however, our study also hints at the involvement of other genes (e.g., Table 7), which might be worth pursuing.

It should be noted that, although we performed RNA sequencing on a precious collection of well-characterized individuals for whom autopsy tissue was available, the actual number of samples included in our study is limited. This mainly affects the clinico-pathological association analyses performed in the subset of individuals carrying an expanded C9orf72 repeat; these analyses, therefore, should be considered exploratory in nature. Additionally, we would like to stress that patients included in this study were generally younger than control subjects. Despite the fact that we adjusted our models for age at death, we realize that this age difference may have influenced our findings. Another limitation that should be mentioned is that we performed RNA sequencing on bulk tissue from the frontal cortex instead of on single nuclei. Because expression levels are cell-type dependent, we included five genes in our models as surrogate markers $[1,12,23]$. Evidently, this approach is not perfect, but it enabled us to (partially) account for various degrees of neuronal loss, inflammation, and gliosis seen in patients with FTLD and/or MND. When taking the cost of single nuclei RNA 
sequencing into consideration, our bulk tissue analysis with adjustment for cellular composition seems to provide a cost-effective alternative that can yield significant results. Future studies could further investigate expression levels of interesting candidates in specific cell types to elucidate which cells are most relevant for a given gene and appear to drive the detected associations (e.g., using purified cell populations), and additionally, they could clarify whether changes on the protein level mirror changes on the RNA level.

\section{Conclusions}

To conclude, in this study, we have used a combination of conventional analyses and machine learning to capture the RNA signature of C9orf72-linked diseases. Our powerful approach highlights the disruptive effects of a repeat expansion in C9orf72, particularly on vesicular transport. Furthermore, we have discovered promising candidate modifiers that were consistently associated with relevant disease features and that may serve as urgently needed biomarkers and/or point to new treatment strategies.

\section{Additional file}

Additional file 1: Figure S1 a Module-trait relationships are presented for patients with an expanded C9orf72 repeat and patients without this repeat (C9Plus vs. C9Minus). b For patients with an expansion and contro subjects (C9Plus vs. Control), module-trait relationships are plotted. These plots are generated with weighted gene co-expression network analysis (WGCNA) to find groups of genes that go up (red) or down (blue) together. A unique color has been assigned to each of these groups, also called a module. Correlations and p-values are shown for variables of interest, including disease group (C9Plus, C9Minus, and/or Control; arrow), neurons, microglia, astrocytes, oligodendrocytes, endothelial cells, RNA integrity number (RIN), age at death, sex, and plate. The strongest correlations (brightest colors) are observed for cell types. Notably, both module-trait relationship plots are based on residuals obtained without adjustment for cell-type-specific markers. Figure S2 a With adjustment for cell-type-specific markers, a cluster dendrogram is shown for C9orf72 expansion carriers and control subjects. b For the same comparison, a cluster dendrogram is displayed without adjustment for cell-type-specific markers. The branches in these dendrograms correspond to specific modules. A unique color has been assigned to each of these modules. Additionally, variables of interest are included, such as the disease group, neurons, microglia, astrocytes, oligodendrocytes, endothelial cells, RNA integrity number (RIN), age at death, sex, and plate. High levels are shown in red and low levels in blue. After adjustment, no striking differences are observed based on cell type; without adjustment, however, modules appear to be associated with certain cell types (e.g., turquoise and neurons). Figure S3 a For patients harboring a C9orf72 repeat expansion and those without this expansion (C9Plus vs. C9Minus; module membership > 0.6 and significance < 1.0E-05), a gene network is displayed. $\mathbf{b}$ A gene network is visualized when examining expansion carriers and controls (C9Plus vs. Control; module membership $>0.6$ and significance $<1.0 \mathrm{E}-05$ ). In these network plots, the connectivity of each gene is represented by the size of its node, the module to which it has been assigned by its color, and the strength of the correlation by the thickness of its edges; the C9orf72 gene is denoted by an arrow. Of note, the plots in this figure have been generated without adjustment for cell-type-specific markers. Figure S4 a-d Trends are displayed for patients carrying a C9orf72 repeat expansion. a The first plot shows VEGFA and age at onset. b CDKL1 and C9orf72 expansion size are shown in the second plot. c The third plot displays EEF2K and poly(GP) levels. In these three plots, the solid blue line denotes the linear regression line, while each individual is represented by a solid dark grey circle. $\mathbf{d}$ The last plot shows SGSM3 levels and survival after onset, when comparing the bottom 50\% (solid salmon line) to the top 50\% (solid turquoise line). These plots have been created using residuals unadjusted for differences in cellular composition. Figure S5 $\mathbf{a}-\mathbf{h}$ The expression levels of VEGFA, CDKL1,EEF2K, and SGSM3 are shown for all disease groups: patients with a C9orf72 repeat expansion (C9Plus), patients without this expansion (C9Minus), and control subjects (Control), both with and without adjustment for cell-type-specific markers. For each box plot, the median is represented by a solid black line, and each box spans the interquartile range (IQR; 25 th percentile to 75 th percentile). Figure $\mathbf{S} \mathbf{6} \mathbf{a}-\mathbf{h}$ This figure displays the correlation between our expression assays (relative expression) and RNA sequencing data (residuals). a-b The first two plots show correlations for VEGFA, either with or without adjustment for cell-type-specific markers. c-d The next two plots visualize correlations for CDKL1, both with and without adjustment for cellular composition. e-f EEF2K is displayed on the next plots, again with and without adjustment for surrogate markers. $\mathbf{g}$ - $\mathbf{h}$ The last two plots show correlations for SGSM3 with and without adjustment for cellular composition. For each of these plots, the solid blue line denotes the linear regression line, while each individual is represented by a solid dark grey circle. (PDF 2894 kb)

\section{Abbreviations}

ALS: Amyotrophic lateral sclerosis; bicor: Biweight midcorrelation; C9orf72: C9orf72-SMCR8 complex subunit; CD34: CD34 molecule; CD68: CD68 molecule; CDKL1: Cyclin dependent kinase like 1; CHMP2B: Charged multivesicular body protein 2B; Cl: Confidence interval; $C Q N$ : Conditional quantile normalization; DCTN6: Dynactin subunit 6; DENN: Differentially expressed in normal and neoplasia; DPR: Dipeptide repeat; EEF2K: Eukaryotic elongation factor 2 kinase; ENO2: Enolase 2; FDR: False discovery rate; FTD: Frontotemporal dementia; FTLD: Frontotemporal lobar degeneration; GEF: GDP/GTP exchange factor; GFAP: Glial fibrillary acidic protein; HR: Hazard ratio; iPSC: Induced pluripotent stem cell; IQR: Interquartile range; LASSO: Least Absolute Shrinkage and Selection Operator; LINE: Long interspersed nuclear element; MND: Motor neuron disease; MSigDB: Molecular signatures database; NT5DC1: 5'-nucleotidase domain containing 1; OLIG2: Oligodendrocyte transcription factor 2; PYCR2: Pyrroline-5-carboxylate reductase 2; RIN: RNA integrity number; RPKM: Reads per kb per million; S100B: S100 calcium binding protein B; SGSM3: Small G protein signaling modulator 3; SMIM14: Small integral membrane protein 14; SOD1: Superoxide dismutase 1; SOV: Source of variation; STAR: Spliced Transcripts Alignment to a Reference; TDP-43: TAR DNA-binding protein 43; VEGFA: Vascular endothelial growth factor A; WGCNA: Weighted gene co-expression network analysis

\section{Acknowledgements}

We thank the Advanced Academic Programs (AAP) and Engineering for Professionals Programs (EPP) at Johns Hopkins University (Baltimore, MD) for their academic support.

\section{Authors' contributions}

DWD, NGR, BFB, RCP, DSK, KAJ, LP, and BO: revising the manuscript for content, including writing of content, contribution of vital reagents/tools/ patients, and obtaining funding. MCB, JL, MDJ, NAF, CP, TFG, and MEM: acquisition of data, analysis or interpretation of data, and revising the manuscript for content, including writing of content. ST, YR, JSR, JWS, and YWA: analysis or interpretation of data and drafting the manuscript for content, including writing of content. MGH: analysis or interpretation of data, statistical analysis or interpretation of data, and drafting the manuscript for content, including writing of content. RR and MVB: study concept or design, acquisition of data, analysis or interpretation of data, statistical analysis or interpretation of data, drafting the manuscript for content, including writing of content, revising the manuscript for content, including writing of content, study supervision or coordination, and obtaining funding. All authors read and approved the final manuscript.

\section{Funding}

The research leading to these results has received funding by the National Institutes of Health (NIH) grants R21 NS110994, R21 NS099631, R35 
NS097261, R35 NS097273, P50 AG016574, P01 NS084974, U01 AG045390, and U54 NS092089 as well as The ALS Association and Robert Packard Center for ALS Research.

\section{Availability of data and materials}

Upon reasonable request, data and/or scripts used for this study will be shared by the corresponding authors.

\section{Ethics approval and consent to participate}

The Brain Bank for Neurodegenerative Disorders at Mayo Clinic operates under IRB ID:15 009452 (Full Title: "Mayo Clinic Florida Brain Bank"). Research on autopsy samples is considered exempt from Human Subjects Research under Federal Regulation 45 CFR 46.101. All autopsies are obtained after consent by the legal next-of-kin or someone legally authorized to make this decision. Autopsies are performed with the explicit assumption that tissue will be used for both diagnostic evaluation and research.

\section{Consent for publication}

Not applicable.

\section{Competing interests}

MDJ and RR hold a patent on methods to screen for the hexanucleotide repeat expansion in the C9orf72 gene. All other authors declare that they have no competing interests.

\section{Author details}

${ }^{1}$ Department of Neuroscience, Mayo Clinic, 4500 San Pablo Road, Jacksonville, FL 32224, USA. ²Department of Health Sciences Research, Mayo Clinic, 200 1st St SW, Rochester, MN 55905, USA. ㄹivision of Biomedical Statistics and Informatics, Mayo Clinic, 4500 San Pablo Road, Jacksonville, FL 32224, USA. ${ }^{4}$ Department of Health Sciences Research, Mayo Clinic, 4500 San Pablo Road, Jacksonville, FL 32224, USA. ${ }^{5}$ Department of Neurology, Mayo Clinic, 4500 San Pablo Road, Jacksonville, FL 32224, USA. 'Department of Neurology, Mayo Clinic, 200 1st St SW, Rochester, MN 55905, USA. ${ }^{7}$ Gianforte School of Computing, Montana State University, 357 Barnard Hall, Bozeman, MT 59717, USA.

Received: 7 August 2019 Accepted: 24 August 2019

Published online: 08 October 2019

\section{References}

1. Allen M, Wang X, Burgess JD, Watzlawik J, Serie DJ, Younkin CS, Nguyen T, Malphrus KG, Lincoln S, Carrasquillo MM et al (2018) Conserved brain myelination networks are altered in Alzheimer's and other neurodegenerative diseases. Alzheimers Dement 14:352-366. https://doi.org/10.1016/j.jalz.2017.09.012

2. Amick J, Roczniak-Ferguson A, Ferguson SM (2016) C9orf72 binds SMCR8, localizes to lysosomes, and regulates mTORC1 signaling. Mol Biol Cell 27: 3040-3051. https://doi.org/10.1091/mbc.E16-01-0003

3. Aoki Y, Manzano R, Lee Y, Dafinca R, Aoki M, Douglas AGL, Varela MA, Sathyaprakash C, Scaber J, Barbagallo P et al (2017) C9orf72 and RAB7L1 regulate vesicle trafficking in amyotrophic lateral sclerosis and frontotemporal dementia. Brain 140:887-897. https://doi.org/10.1093/brain/awx024

4. Ash PE, Bieniek KF, Gendron TF, Caulfield T, Lin WL, Dejesus-Hernandez M, van Blitterswijk MM, Jansen-West K, Paul JW 3rd, Rademakers R et al (2013) Unconventional translation of C9ORF72 GGGGCC expansion generates insoluble polypeptides specific to c9FTD/ALS. Neuron 77:639-646. https://doi.org/10.1016/j.neuron.2013.02.004

5. Benjamini $Y$, Hochberg $Y$ (1995) Controlling the false discovery rate: a practical and powerful approach to multiple testing. J R Stat Soc Ser B Methodol 57:289-300

6. Breiman L (2001) Random forests. Mach Learn 45:5-32. https://doi.org/10.1 023/a:1010933404324

7. Burrell JR, Kiernan MC, Vucic S, Hodges JR (2011) Motor neuron dysfunction in frontotemporal dementia. Brain 134:2582-2594. https://doi.org/10.1093/ brain/awr195

8. Canning P, Park K, Goncalves J, Li C, Howard CJ, Sharpe TD, Holt LJ, Pelletier L, Bullock AN, Leroux MR (2018) CDKL family kinases have evolved distinct structural features and ciliary function. Cell Rep 22:885-894. https://doi.org/1 0.1016/j.celrep.2017.12.083
9. Chai N, Gitler AD (2018) Yeast screen for modifiers of C9orf72 poly (glycinearginine) dipeptide repeat toxicity. FEMS Yeast Res 18. https://doi.org/10.1 093/femsyr/foy024

10. Chen H (2018) VennDiagram: Generate High-Resolution Venn and Euler Plots. R Package Version 1.6.20

11. Cooper-Knock J, Bury JJ, Heath PR, Wyles M, Higginbottom A, Gelsthorpe C, Highley JR, Hautbergue G, Rattray M, Kirby J et al (2015) C9ORF72 GGGGCC expanded repeats produce splicing dysregulation which correlates with disease severity in amyotrophic lateral sclerosis. PLoS One 10:e0127376. https://doi.org/10.1371/journal.pone.0127376

12. De Jager PL, Srivastava G, Lunnon K, Burgess J, Schalkwyk LC, Yu L, Eaton ML, Keenan BT, Ernst J, McCabe C et al (2014) Alzheimer's disease: early alterations in brain DNA methylation at ANK1, BIN1, RHBDF2 and other loci. Nat Neurosci 17:1156-1163. https://doi.org/10.1038/nn.3786

13. DeJesus-Hernandez M, Finch NA, Wang X, Gendron TF, Bieniek KF, Heckman MG, Vasilevich A, Murray ME, Rousseau L, Weesner R et al (2017) In-depth clinico-pathological examination of RNA foci in a large cohort of C9ORF72 expansion carriers. Acta Neuropathol 134:255-269. https://doi.org/10.1007/ s00401-017-1725-7

14. DeJesus-Hernandez M, Mackenzie IR, Boeve BF, Boxer AL, Baker M, Rutherford NJ, Nicholson AM, Finch NA, Flynn H, Adamson J et al (2011) Expanded GGGGCC hexanucleotide repeat in noncoding region of C9ORF72 causes chromosome 9p-linked FTD and ALS. Neuron 72:245-256. https://doi.org/10.1016/j.neuron.2011.09.011

15. Dobin A, Davis CA, Schlesinger F, Drenkow J, Zaleski C, Jha S, Batut P, Chaisson M, Gingeras TR (2013) STAR: ultrafast universal RNA-seq aligner. Bioinformatics 29:15-21. https://doi.org/10.1093/bioinformatics/bts635

16. Donnelly CJ, Zhang PW, Pham JT, Haeusler AR, Mistry NA, Vidensky S, Daley EL, Poth EM, Hoover B, Fines DM et al (2013) RNA toxicity from the ALS/FTD C9ORF72 expansion is mitigated by antisense intervention. Neuron 80:415428. https://doi.org/10.1016/j.neuron.2013.10.015

17. Elamin M, Bede P, Byrne S, Jordan N, Gallagher L, Wynne B, O'Brien C, Phukan J, Lynch C, Pender N et al (2013) Cognitive changes predict functional decline in ALS: a population-based longitudinal study. Neurology 80:1590-1597. https://doi.org/10.1212/WNL.0b013e31828f18ac

18. Farg MA, Sundaramoorthy V, Sultana JM, Yang S, Atkinson RA, Levina V, Halloran MA, Gleeson PA, Blair IP, Soo KY et al (2014) C9ORF72, implicated in amytrophic lateral sclerosis and frontotemporal dementia, regulates endosomal trafficking. Hum Mol Genet 23:3579-3595. https://doi.org/10.1093/hmg/ddu068

19. Finch NA, Wang X, Baker MC, Heckman MG, Gendron TF, Bieniek KF, Wuu J, DeJesus-Hernandez M, Brown PH, Chew J et al (2017) Abnormal expression of homeobox genes and transthyretin in C9ORF72 expansion carriers. Neurol Genet 3:e161. https://doi.org/10.1212/NXG.0000000000000161

20. Friedman J, Hastie T, Tibshirani R (2010) Regularization paths for generalized linear models via coordinate descent. J Stat Softw 33:1-22

21. Gendron TF, van Blitterswijk M, Bieniek KF, Daughrity LM, Jiang J, Rush BK, Pedraza O, Lucas JA, Murray ME, Desaro P et al (2015) Cerebellar c9RAN proteins associate with clinical and neuropathological characteristics of C9ORF72 repeat expansion carriers. Acta Neuropathol 130:559-573. https://doi.org/10.1007/s00401-015-1474-4

22. Gu Z, Eils R, Schlesner M (2016) Complex heatmaps reveal patterns and correlations in multidimensional genomic data. Bioinformatics 32:28472849. https://doi.org/10.1093/bioinformatics/btw313

23. Gusareva ES, Carrasquillo MM, Bellenguez C, Cuyvers E, Colon S, GraffRadford NR, Petersen RC, Dickson DW, Mahachie John JM, Bessonov K et al (2014) Genome-wide association interaction analysis for Alzheimer's disease. Neurobiol Aging 35:2436-2443. https://doi.org/10.1016/j.neurobiolaging.2 014.05.014

24. Hansen KD, Irizarry RA, Wu Z (2012) Removing technical variability in RNAseq data using conditional quantile normalization. Biostatistics 13:204-216. https://doi.org/10.1093/biostatistics/kxr054

25. Heise C, Gardoni F, Culotta L, di Luca M, Verpelli C, Sala C (2014) Elongation factor-2 phosphorylation in dendrites and the regulation of dendritic mRNA translation in neurons. Front Cell Neurosci 8:35. https://doi.org/10.3389/ fncel.2014.00035

26. Jan A, Jansonius B, Delaidelli A, Bhanshali F, An YA, Ferreira N, Smits LM, Negri GL, Schwamborn JC, Jensen PH et al (2018) Activity of translation regulator eukaryotic elongation factor-2 kinase is increased in Parkinson disease brain and its inhibition reduces alpha synuclein toxicity. Acta Neuropathol Commun 6:54. https://doi.org/10.1186/s40478018-0554-9 
27. Jan A, Jansonius B, Delaidelli A, Somasekharan SP, Bhanshali F, Vandal M, Negri GL, Moerman D, MacKenzie I, Calon F et al (2017) eEF2K inhibition blocks Abeta42 neurotoxicity by promoting an NRF2 antioxidant response. Acta Neuropathol 133:101-119. https://doi.org/10.1007/s00401-016-1634-1

28. Jin K, Zhu Y, Sun Y, Mao XO, Xie L, Greenberg DA (2002) Vascular endothelial growth factor (VEGF) stimulates neurogenesis in vitro and in vivo. Proc Natl Acad Sci U S A 99:11946-11950. https://doi.org/10.1073/ pnas.182296499

29. Keifer OP Jr, O'Connor DM, Boulis NM (2014) Gene and protein therapies utilizing VEGF for ALS. Pharmacol Ther 141:261-271. https://doi.org/10.1016/ j.pharmthera.2013.10.009

30. Lagier-Tourenne C, Baughn M, Rigo F, Sun S, Liu P, Li HR, Jiang J, Watt AT, Chun S, Katz M et al (2013) Targeted degradation of sense and antisense C9orf72 RNA foci as therapy for ALS and frontotemporal degeneration. Proc Natl Acad Sci U S A 110:E4530-E4539. https://doi.org/10.1073/pnas.1318835110

31. Lambrechts D, Poesen K, Fernandez-Santiago R, Al-Chalabi A, Del Bo R, Van Vught PW, Khan S, Marklund SL, Brockington A, van Marion I et al (2009) Meta-analysis of vascular endothelial growth factor variations in amyotrophic lateral sclerosis: increased susceptibility in male carriers of the -2578AA genotype. J Med Genet 46:840-846. https://doi.org/10.1136/jmg.2008.058222

32. Lambrechts D, Storkebaum E, Morimoto M, Del-Favero J, Desmet F, Marklund SL, Wyns S, Thijs V, Andersson J, van Marion I et al (2003) VEGF is a modifier of amyotrophic lateral sclerosis in mice and humans and protects motoneurons against ischemic death. Nat Genet 34:383-394. https://doi.org/10.1038/ng1211

33. Langfelder P (2018) anRichment: collections and annotation data for use with anRichmentMethods. $\mathrm{R}$ package version 0.97-1

34. Langfelder P, Horvath S (2008) WGCNA: an R package for weighted correlation network analysis. BMC Bioinf 9:559. https://doi.org/10.1186/14 71-2105-9-559

35. Langfelder P, Horvath S (2012) Fast R functions for robust correlations and hierarchical clustering. J Stat Softw 46:i11

36. Levine TP, Daniels RD, Gatta AT, Wong LH, Hayes MJ (2013) The product of C9orf72, a gene strongly implicated in neurodegeneration, is structurally related to DENN Rab-GEFs. Bioinformatics 29:499-503. https://doi.org/10.1 093/bioinformatics/bts 725

37. Liao Y, Smyth GK, Shi W (2014) featureCounts: an efficient general purpose program for assigning sequence reads to genomic features. Bioinformatics 30:923-930. https://doi.org/10.1093/bioinformatics/btt656

38. Liaw A, Wiener M (2002) Classification and regression by randomForest. $R$ News 2(3):18-22

39. Liberzon A, Subramanian A, Pinchback R, Thorvaldsdottir H, Tamayo P, Mesirov JP (2011) Molecular signatures database (MSigDB) 3.0. Bioinformatics 27:1739-1740. https://doi.org/10.1093/bioinformatics/btr260

40. Liu EY, Russ J, Wu K, Neal D, Suh E, McNally AG, Irwin DJ, Van Deerlin VM, Lee EB (2014) C9orf72 hypermethylation protects against repeat expansionassociated pathology in ALS/FTD. Acta Neuropathol 128:525-541. https:// doi.org/10.1007/s00401-014-1286-y

41. Majounie E, Renton AE, Mok K, Dopper EG, Waite A, Rollinson S, Chio A Restagno G, Nicolaou N, Simon-Sanchez J et al (2012) Frequency of the C9orf72 hexanucleotide repeat expansion in patients with amyotrophic lateral sclerosis and frontotemporal dementia: a cross-sectional study. Lancet Neurol 11:323-330. https://doi.org/10.1016/S1474-4422(12)70043-1

42. Mori K, Weng SM, Arzberger T, May S, Rentzsch K, Kremmer E, Schmid B, Kretzschmar HA, Cruts M, Van Broeckhoven C et al (2013) The C9orf72 GGGGCC repeat is translated into aggregating dipeptide-repeat proteins in FTLD/ALS. Science 339:1335-1338. https://doi.org/10.1126/science.1232927

43. Neumann M, Sampathu DM, Kwong LK, Truax AC, Micsenyi MC, Chou TT, Bruce J, Schuck T, Grossman M, Clark CM et al (2006) Ubiquitinated TDP-43 in frontotemporal lobar degeneration and amyotrophic lateral sclerosis. Science 314:130-133. https://doi.org/10.1126/science.1134108

44. Nguyen HP, Van Broeckhoven C, van der Zee J (2018) ALS genes in the genomic era and their implications for FTD. Trends Genet 34:404-423. https://doi.org/10.1016/j.tig.2018.03.001

45. Oosthuyse B, Moons L, Storkebaum E, Beck H, Nuyens D, Brusselmans K, Van Dorpe J, Hellings P, Gorselink M, Heymans S et al (2001) Deletion of the hypoxia-response element in the vascular endothelial growth factor promoter causes motor neuron degeneration. Nat Genet 28:131-138. https://doi.org/10.1038/88842

46. Phukan J, Elamin M, Bede P, Jordan N, Gallagher L, Byrne S, Lynch C, Pender N, Hardiman O (2012) The syndrome of cognitive impairment in amyotrophic lateral sclerosis: a population-based study. J Neurol Neurosurg Psychiatry 83:102-108. https://doi.org/10.1136/jnnp-2011-300188

47. Prudencio M, Belzil W, Batra R, Ross CA, Gendron TF, Pregent LJ, Murray ME, Overstreet KK, Piazza-Johnston AE, Desaro P et al (2015) Distinct brain transcriptome profiles in C9orf72-associated and sporadic ALS. Nat Neurosci 18:1175-1182. https://doi.org/10.1038/nn.4065

48. Prudencio M, Gonzales PK, Cook CN, Gendron TF, Daughrity LM, Song Y, Ebbert MTW, van Blitterswijk M, Zhang YJ, Jansen-West K et al (2017) Repetitive element transcripts are elevated in the brain of C9orf72 ALS/FTLD patients. Hum Mol Genet 26:3421-3431. https://doi.org/10.1093/hmg/ddx233

49. Ren $Y$, van Blitterswijk M, Allen M, Carrasquillo MM, Reddy JS, Wang X, Beach TG, Dickson DW, Ertekin-Taner N, Asmann YW et al (2018) TMEM106B haplotypes have distinct gene expression patterns in aged brain. Mol Neurodegener 13:35. https://doi.org/10.1186/s13024-018-0268-2

50. Renton AE, Majounie E, Waite A, Simon-Sanchez J, Rollinson S, Gibbs JR, Schymick JC, Laaksovirta H, van Swieten JC, Myllykangas L et al (2011) A hexanucleotide repeat expansion in C9ORF72 is the cause of chromosome 9p21-linked ALS-FTD. Neuron 72:257-268. https://doi.org/10.1016/j.neuron.2 011.09 .010

51. Russ J, Liu EY, Wu K, Neal D, Suh E, Irwin DJ, McMillan CT, Harms MB, Cairns NJ. Wood EM et al (2015) Hypermethylation of repeat expanded C9orf72 is a clinical and molecular disease modifier. Acta Neuropathol 129:39-52. https://doi.org/10.1007/s00401-014-1365-0

52. Sareen D, O'Rourke JG, Meera P, Muhammad AK, Grant S, Simpkinson M, Bell S, Carmona S, Ornelas L, Sahabian A et al (2013) Targeting RNA foci in iPSCderived motor neurons from ALS patients with a C9ORF72 repeat expansion. Sci Transl Med 5:208ra149. https://doi.org/10.1126/scitranslmed.3007529

53. Sellier C, Campanari ML, Julie Corbier C, Gaucherot A, Kolb-Cheynel I, OuladAbdelghani M, Ruffenach F, Page A, Ciura S, Kabashi E et al (2016) Loss of C9ORF72 impairs autophagy and synergizes with polyQ Ataxin-2 to induce motor neuron dysfunction and cell death. EMBO J 35:1276-1297. https://doi.org/10.15252/embj.201593350

54. Selvaraj BT, Livesey MR, Zhao C, Gregory JM, James OT, Cleary EM, Chouhan AK, Gane AB, Perkins EM, Dando O et al (2018) C9ORF72 repeat expansion causes vulnerability of motor neurons to ca (2+)-permeable AMPA receptormediated excitotoxicity. Nat Commun 9:347. https://doi.org/10.1038/s41467017-02729-0

55. Shannon P, Markiel A, Ozier O, Baliga NS, Wang JT, Ramage D, Amin N, Schwikowski B, Ideker T (2003) Cytoscape: a software environment for integrated models of biomolecular interaction networks. Genome Res 13: 2498-2504. https://doi.org/10.1101/gr.1239303

56. Shi Y, Lin S, Staats KA, Li Y, Chang WH, Hung ST, Hendricks E, Linares GR, Wang Y, Son EY et al (2018) Haploinsufficiency leads to neurodegeneration in C9ORF72 ALS/FTD human induced motor neurons. Nat Med 24:313-325. https://doi.org/10.1038/nm.4490

57. van Blitterswijk M, Dejesus-Hernandez M, Niemantsverdriet E, Murray ME, Heckman MG, Diehl NN, Brown PH, Baker MC, Finch NA, Bauer PO et al (2013) Association between repeat sizes and clinical and pathological characteristics in carriers of C9ORF72 repeat expansions (Xpansize-72): a cross-sectional cohort study. Lancet Neurol 12:978-988. https://doi.org/10.1 016/S1474-4422(13)70210-2

58. van Blitterswijk M, DeJesus-Hernandez M, Rademakers R (2012) How do C9ORF72 repeat expansions cause amyotrophic lateral sclerosis and frontotemporal dementia: can we learn from other noncoding repeat expansion disorders? Curr Opin Neurol 25:689-700. https://doi.org/10.1097/ WCO.0b013e32835a3efb

59. van Blitterswijk M, Gendron TF, Baker MC, DeJesus-Hernandez M, Finch NA, Brown PH, Daughrity LM, Murray ME, Heckman MG, Jiang J et al (2015) Novel clinical associations with specific C9ORF72 transcripts in patients with repeat expansions in C9ORF72. Acta Neuropathol 130:863-876. https://doi. org/10.1007/s00401-015-1480-6

60. Wang L, Wang S, Li W (2012) RSeQC: quality control of RNA-seg experiments. Bioinformatics 28:2184-2185. https://doi.org/10.1093/ bioinformatics/bts356

61. Wang X, Dalkic E, Wu M, Chan C (2008) Gene module level analysis: identification to networks and dynamics. Curr Opin Biotechnol 19:482-491. https://doi.org/10.1016/j.copbio.2008.07.011

62. Webster CP, Smith EF, Bauer CS, Moller A, Hautberque GM, Ferraiuolo L, Myszczynska MA, Higginbottom A, Walsh MJ, Whitworth AJ et al (2016) The C9orf72 protein interacts with Rabla and the ULK1 complex to regulate initiation of autophagy. EMBO J 35:1656-1676. https://doi.org/10.15252/embj.201694401 
63. Yang H, Sasaki T, Minoshima S, Shimizu N (2007) Identification of three novel proteins (SGSM1, 2, 3) which modulate small G protein (RAP and RAB)-mediated signaling pathway. Genomics 90:249-260. https://doi.org/1 0.1016/j.ygeno.2007.03.013

64. Yang M, Liang C, Swaminathan K, Herrlinger S, Lai F, Shiekhattar R, Chen JF (2016) A C9ORF72/SMCR8-containing complex regulates ULK1 and plays a dual role in autophagy. Sci Adv 2:e1601167. https://doi. org/10.1126/sciadv.1601167

65. Zhang D, lyer LM, He F, Aravind L (2012) Discovery of novel DENN proteins: implications for the evolution of eukaryotic intracellular membrane structures and human disease. Front Genet 3:283. https://doi.org/10.3389/ fgene. 2012.00283

\section{Publisher's Note}

Springer Nature remains neutral with regard to jurisdictional claims in published maps and institutional affiliations.

Ready to submit your research? Choose BMC and benefit from:

- fast, convenient online submission

- thorough peer review by experienced researchers in your field

- rapid publication on acceptance

- support for research data, including large and complex data types

- gold Open Access which fosters wider collaboration and increased citations

- maximum visibility for your research: over $100 \mathrm{M}$ website views per year

At $\mathrm{BMC}$, research is always in progress.

Learn more biomedcentral.com/submissions 